\title{
Nobel Lecture: Cosmic microwave background radiation anisotropies: Their discovery and utilization*
}

\author{
George F. Smoot \\ Lawrence Berkeley National Laboratory, Space Sciences Laboratory, Department of \\ Physics, University of California, Berkeley, California 94720, USA
}

(Published 2 November 2007)

DOI: $10.1103 /$ RevModPhys.79.1349

\section{THE COSMIC BACKGROUND RADIATION}

Observations of the cosmic microwave background (CMB) temperature anisotropies have revolutionized and continue to revolutionize our understanding of the universe. The observation of the CMB anisotropies angular power spectrum with its plateau, acoustic peaks, and high frequency damping tail have established a standard cosmological model consisting of a flat (critical density) geometry, with contents being mainly dark energy and dark matter and a small amount of ordinary matter. In this successful model the dark and ordinary matter formed its structure through gravitational instability acting on the quantum fluctuations generated during the very early inflationary epoch. Current and future observations will test this model and determine its key cosmological parameters with spectacular precision and confidence.

\section{A. Introduction}

In the big bang theory the cosmic microwave background $(\mathrm{CMB})$ radiation is the relic radiation from the hot primeval fireball that began our observable universe about 13.7 billion years ago. As such the CMB can be used as a powerful tool that allows us to measure the dynamics and geometry of the universe. The CMB was first discovered by Penzias and Wilson at Bell Laboratory in 1964 (Penzias and Wilson, 1965). They found a persistent radiation from every direction which had a thermodynamic temperature of about $3.2 \mathrm{~K}$. At that time, physicists at Princeton (Dicke, 1965; Dicke et al., 1965) were developing an experiment to measure the relic radiation from the big bang theory. Penzias and Wilson's serendipitous discovery of the CMB opened up the new era of cosmology, beginning the process of transforming it from myth and speculation into a real scientific exploration. According to big bang theory, our universe began in a nearly perfect thermal equilibrium state with very high temperature. The universe is dynamic and has been ever expanding and cooling since its birth. When the temperature of the universe dropped to

\footnotetext{
*The 2006 Nobel Prize for Physics was shared by John C. Mather and George F. Smoot. This paper is the text of the address given in conjunction with the award.
}

$3000 \mathrm{~K}$ there were insufficient energetic CMB photons to keep hydrogen or helium atoms ionized. Thus the primeval plasma of charged nuclei, electrons, and photons changed into neutral atoms plus background radiation. The background radiation could then propagate through space freely, though being stretched by the continuing expansion of the universe, while baryonic matter (mostly hydrogen and helium atoms) could cluster by gravitational attraction to form stars, galaxies, and even larger structures. For these structures to form there must have been primordial perturbations in the early matter and energy distributions. The primordial fluctuations of matter density that will later form large scale structures leave imprints in the form of temperature anisotropies in the $\mathrm{CMB}$.

\section{B. Cosmic background radiation rules}

As a young undergraduate I heard of Penzias and Wilson's (1965) discovery of the $3 \mathrm{~K}$ background radiation and its interpretation by Dicke, Peebles, Roll, and Wilkingon (1965), but not until two or three years later did I begin to understand the implications and opportunity it afforded. I was a first year graduate student at MIT working on a high-energy physics experiment when Joe Silk, then a graduate student at nearby Harvard, published a paper (Silk, 1967) entitled "Fluctuations in the Primordial Fireball" with the abstract "One of the overwhelming difficulties of realistic cosmological models is the inadequacy of Einstein's gravitational theory to explain the process of galaxy formation. ${ }^{16} \mathrm{~A}$ means of evading this problem has been to postulate an initial spectrum of primordial fluctuations. ${ }^{7}$ The interpretation of the recently discovered $3{ }^{\circ} \mathrm{K}$ microwave background as being of cosmological origin ${ }^{8,9}$ implies that fluctuations may not condense out of the expanding universe until an epoch when matter and radiation have decoupled, ${ }^{4}$ at a temperature $T_{D}$ of the order of $4000{ }^{\circ} \mathrm{K}$. The question may then be posed: would fluctuations in the primordial fireball survive to an epoch when galaxy formation is possible?"

My physics colleagues dismissed this work as speculation and not a real scientific enquiry. It seemed to me a field ripe for observations that would be important no matter how they came out. Obviously, there were galaxies. Determining if the radiation was cosmic was critical. 
If the $3 \mathrm{~K}$ microwave background was cosmic, it must contain imprints of fluctuations from a very early epoch when energies were very high. Silk's work also made me realize the enormously important role of the cosmic background radiation in the early universe. Going back to earlier times when the universe was smaller, one would reach the epoch when the radiation was as bright as the sun. At this epoch the universe was roughly a thousand times smaller than present. This is impressively small but one could readily and reasonably extrapolate back another thousand in size and then the radiation would be a thousand times hotter than the sun. ${ }^{1}$

But in truth, if this was the relic radiation, then the pioneering calculations of Gamow and co-workers (Alpher, Bethe, and Gamow, 1948; Alpher, Herman, and Gamow, 1948; Alpher and Herman, 1953, 1988, 1990; Alpher et al., 1953, 1967) tell us we can comfortably and reliably look back to the point where the universe was a billion $\left(10^{9}\right)$ times smaller. This is the epoch of primordial nucleosynthesis when the first nuclei form and their calculations correctly predicted the ratio of hydrogen to helium and the abundance of a few light elements. At that epoch the temperature of the radiation was a million times greater (and $10^{24}$ times brighter) than that of the sun. Any object placed in that radiation bath would be nearly instantly vaporized and homogenized. Even atoms were stripped apart. At such early times the nuclei of atoms would be blown apart. The very early universe had to exist in a very simple state completely dominated by the cosmic background radiation which would tear everything into its simplest constituents and spread it uniformly about.

Also in 1967 Dennis Sciama published a paper (Sciama, 1967) pointing out that if this were relic radiation from the big bang, one could test Mach's principle and measure the rotation of the universe by the effect that rotation would have on the cosmic microwave background. It could rule out Godel's model of a rotating universe and its implied time travel supporting Mach's principle and keeping us safe from time tourists. Here was another fundamental physics and potentially exciting observation that one could make, if the $\mathrm{CMB}$ were cosmological in origin.

Not long after (submitted October 1967, published April 1968) Stephen W. Hawking and George F. R. Ellis published a paper "The cosmic black-body radiation and the existence of singularities in our universe" (Hawking and Ellis, 1968) which used the early singularity theo-

\footnotetext{
${ }^{1}$ If the radiance of a thousand suns

were to burst into the sky,

that would be like

the splendor of the Mighty One
}

I am become Death, the shatterer of Worlds.

reported J. Robert Oppenheimer quote of the Gita at the first atomic bomb test 16 July 1945. Since the Gita's first translation into English in 1785, most experts have translated not "Death" but instead "Time." The atomic fireball when first visible would roughly be a thousand times the temperature of the sun. rems of Penrose, Hawking, and Geroch to show that if the $\mathrm{CMB}$ was the relic radiation of the big bang, and if it were observed to be isotropic to a high degree, e.g., a part in 100, that one could not avoid having a singularity in the early universe. The rough argument goes that, if the $\mathrm{CMB}$ is cosmological and uniform to a high level, say one part in $X$, then one could extrapolate the universe backwards to a time when it was $1 / X$ smaller. If $X$ is sufficiently large, then the energy density in the CBR (microwaves now much more intense and hotter) would be sufficient to close the universe and cause it to extrapolate right back to the singularity. The only premises in the argument were (i) the CMB was cosmological, (ii) it would be found to be uniform to about a part in 10000 ( $X=100$ in their original optimistic argument but actually 10000 in present understanding), (iii) general relativity or a geometric theory of gravity are the correct description, and (iv) the energy condition that there is no substance which has negative energy densities or large negative pressures. Hawking and Ellis provided strongly plausible arguments against violation of the energy condition. This observation would certainly be a death blow to the numerous popular oscillating universe models and other attempts to make models without a primordial singularity. Once again we see theorists providing arguments for the cosmic implications that could be drawn from observations of the $\mathrm{CMB}$-if it were truly cosmological.

One needed to be of two minds about the CMB: (i) be skeptical and test carefully to see that it was not the relic radiation of the big bang and (ii) assume that it was the relic radiation and had the properties expected and then look for the small deviations and thus information that it could reveal about the universe. Early on one had to make a lot of assumptions about the CMB in order to use it as a tool to probe the early universe, but as more and more observations have been made and care taken, these assumptions have been tested and probed more and more precisely and fully. The history of the observations and theoretical developments is rife with this approach. The discovery of the CMB by Penzias and Wilson was serendipitous. They came upon it without having set out to find it or even to explore for some new thing. In retrospect the discovery, though serendipitous, was not in a vacuum. There were ideas back to the time of Gamow (Alpher, Bethe, and Gamow, 1948; Alpher, Herman, and Gamow, 1948; Alpher and Herman, 1953, 1988, 1990; Alpher et al., 1953, 1967), Doroshkevich and Novikov (1964), reinvented by Dicke and Peebles (1965) that there should be a relic radiation. There were plenty of observations that in retrospect pointed that there was something there, e.g., McKellar's 1941 observations of the anomalous temperature of $\mathrm{CN}$ molecules in cold clouds, followed by a string of others having noticed something unusual. However, Penzias and Wilson made the definitive observations in the sense that they observed a signal, checked for potential errors, added calibrations, and otherwise made their case air-tight so that the world took notice. 
This tremendously important observation was rapidly interpreted and then a number of theorists began to work out the possibilities and potential implications and make these known to possible observers. Observers, and often their funding sources, who have to invest a significant amount of effort, time, and resources, like to have some assurance that the observations are likely to prove worthwhile.

I immediately understood that what we can actually observe of the relic radiation is its electric field $\vec{E}(v, \theta, \phi, t)$ (or magnetic field $\vec{B}$ ), so I made a table in textbook fashion of the various things one could measure about the radiation based on observing the electric field here and now. My idea was to check each of these in a systematic way to establish clearly that the $\mathrm{CMB}$ was or was not the relic radiation from the big bang and then find out what it could tell us about the early universe. First is the frequency $v$ spectrum of the radiation. If the $3 \mathrm{~K}$ radiation were truly the relic radiation from the early hot universe in thermal equilibrium, then it would have the famous blackbody spectrum whose careful formulation by Max Planck in 1900 initiated quantum theory:

$$
\begin{aligned}
& \bar{n}=\frac{1}{e^{h v / k T}-1}, \quad B(v)=\frac{8 \pi h v^{3}}{c^{2}} \frac{d v}{e^{h v / k T}-1}, \\
& B(\lambda)=\frac{8 \pi h c^{2}}{\lambda^{5}} \frac{d \lambda}{e^{h c / \lambda k T}-1},
\end{aligned}
$$

where $\bar{n}$ is the mean photon occupation number per quantum state and $B(v)$ [and $B(\lambda)]$ is the brightness in units of energy per unit area per second per unit bandwidth (per unit wavelength). This spectrum has the property that it is precisely well prescribed by only one parameter, its temperature $T_{\mathrm{CBR}}$. The demonstration that this was likely to be true took years of effort with many misleading results along the way. The theory of potential slight distortions from the blackbody shape and what that might reveal also took time to develop and be absorbed by observers.

Likewise, one could map the incoming radiation as a function of position on the sky designated by the angles $\theta$ and $\phi$. In the simplest possible big bang model, the relic radiation would be isotropic, that is, independent of the angles $\theta$ and $\phi$ on the sky. To first order, as Penzias and Wilson had shown, the $3 \mathrm{~K}$ radiation was isotropic, but as Silk (1967), Sachs and Wolfe (1967), and others pointed out, there must be some residual perturbations to give rise to galaxies and clusters of galaxies and they give rise to temperature fluctuations across the sky. In these earliest days the fluctuations were anticipated to be fairly large (slightly below the $10 \%$ level limit by Penzias and Wilson) but after careful study they were predicted to be at the one part in a thousand level $(\Delta T / T$ $\sim 10^{-3}$ ). Later the theoretical predictions were to get much smaller.

The vector direction plane of the oscillating electric field $\vec{E}$ is expected to be completely random from purely thermal radiation of a universe in complete thermal equilibrium and high opacity. However, in 1968 Martin Rees (1968) pointed out that small temperature fluctuations and Thompson scattering at the last scattering surface would give rise to a very slight linear polarization of the $\mathrm{CMB}$.

The time dependence $t$ of the electric field $\vec{E}(t)$ shows up in two ways. The first way is that thermal radiation has not only a well-defined distribution but also a welldefined statistical fluctuation spectrum. Specifically, the variance of the number of photons per unit mode $n$ due to the thermal statistical fluctuations should be of the form

$$
\left\langle n^{2}-\bar{n}^{2}\right\rangle=\bar{n}^{2}+\bar{n},
$$

where the first term is called the wave noise and the second term is called the shot noise of individual photons. At low frequencies $h v \ll k_{B} T$ (Rayleigh-Jeans regime) then the wave noise dominates and the rms fluctuations are simply $\bar{n}=1 /\left(e^{h v / k_{B} T}-1\right) \simeq k_{B} T / h v$. The rms fluctuations are proportional to the temperature $T$. At high frequencies $h v \gg k_{B} T$ (the Wien tail), the shot noise dominates. This is a phenomenon that my group tested at low frequencies using correlation radiometers in the 1970s. Likewise, the early bolometer experiments tested the other regime indirectly and this is an assumption that continues forward in present observations, particularly those near the CMB peak where both effects are significant.

There is another second order effect in the correlations of the photons first made manifest in the HanburyBrown and Twiss interferometer and, though tested, it is not so central to CMB observations.

The second time dependence is that as one were to observe the radiation in the distant past, its temperature should increase in direct inverse to the scale size of the universe: $a$ (then) $T_{\text {then }}=a($ now $) T_{\text {now }}$ or $T_{\text {then }}=(1+z) T_{\text {now }}$, where $1+z=a($ now $) / a$ (then) with $a$ being the scale size of the universe at the epochs of interest. This is simply the stretching of wavelengths with the scale change of the universe combined with the Planck law. A number of groups have done experiments to check this dependence and found reasonable but so far limited evidence that supports this dependence. There is abundant evidence that the CMB is not a local phenomenon in that otherwise cold dense molecular clouds in our galaxy and nearby galaxies show additional excitation which just matches the energy input from the CMB. However, as we and others have found out, it is more difficult to make these observations in very distant galaxies (see Table I and Fig. 1).

In taking into account the real universe with real galaxies and clusters of galaxies, there was another probe of the fact that the CMB fills the universe and another eventual cosmological probe with it. In 1970 and more explicitly in 1972, Rashid Sunyaev and Yacob B. Zel'dovich $(1970,1972)$ predicted that the hot ionized medium in galactic clusters provided sufficient free electrons to scatter a small percentage of the CBR photons 
TABLE I. The temperature of the cosmic background radiation for a few redshifts $z$. Values of the $\mathrm{CMB}$ temperature from the observation of the fine-structure transition of the $\mathrm{C} \mathrm{I}$ and $\mathrm{C} \mathrm{II.}$

\begin{tabular}{lcccc}
\hline \hline$z$ & $T(\mathrm{~K})$ & Molecule & Quasar & Reference \\
\hline 1.776 & $<16 @ 2 \sigma$ & C I & QSO 1331+170 & Meyer et al. $(1986)$ \\
1.776 & $7.4 \pm 0.8$ & C I & QSO 1331+170 & Songaila et al. $(1994 \mathrm{~b})$ \\
1.9731 & $7.9 \pm 1.0$ & C I & QSO 0013-004 & Ge et al. $(1997)$ \\
2.309 & $<45 K @ 2 \sigma$ & C II & PHL 957 & Bahcall et al. $(1973)$ \\
2.909 & $<13.5 K @ 2 \sigma$ & C II & QSO 0636+680 & Songaila et al. $(1994 \mathrm{a})$ \\
4.3829 & $<19.6 K @ 3 \sigma$ & C II & QSO 1202-07 & Lu et al. $(1995)$ \\
\hline \hline
\end{tabular}

passing through the cluster. On average, since the electrons were hotter than the CBR photons, they would scatter the photons preferentially to higher frequencies causing a diminution of photons at low frequencies and a surplus at high frequencies. This meant that a cluster of galaxies would cast a faint shadow at low frequencies and glow at higher frequencies, since the CBR photons would come from the greatest possible distances. It was also clear that this was a spectral effect and would be independent of redshift and could be used to observe galaxy clusters across the full observable universe. In 1974, to look for the Sunyaev-Zel'dovich (SZ) effect, Rich Muller and I went to use the Goldstone radiotelescope and its new maser receiver, a key part of NASA's Deep Space Net to observe the Coma cluster (match of beam size and low frequency of observation). Unfortunately, the observations were not quite sufficient to make the detection. However, Mark Birkinshaw (1999) and others continued to pioneer these observations over the next two decades, improving the approach and level of detection. A significant breakthrough came with the use of the Hat Creek Observatory by Carlstrom et al. (2000) with clean high signal-to-noise observations of galaxy clusters showing the expected effect and correlation with $\mathrm{x}$-ray observations. These established without a doubt that the CMB fills the universe and comes from

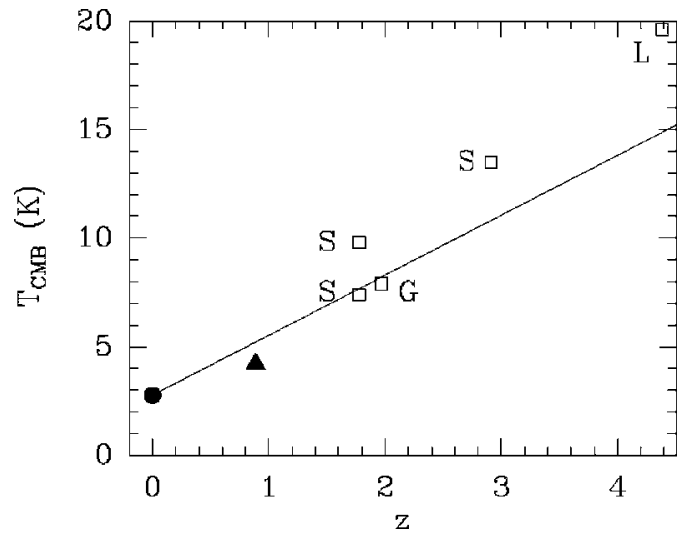

FIG. 1. Summary of CMB temperature measurements as a function of redshift. The filled dot is from COBE (Mather et al. 1994). The squares are upper limits obtained on the C I or C II from Songaila et al. (1994a, 1994b; S), Lu et al. (1995; L), and Ge et al. (1997; G). Combes et al. is the filled triangle. The line is the $(1+z)$ expected variation. From Combes et al., 1999. far beyond the most remote galactic clusters observed. We are soon to see a substantial step forward in the utilization of the SZ effect beginning in 2007 with the observations from new instruments such as APEX-SZ and the South Pole Telescope (2007) (SPT).

\section{Transition to cosmology}

Though intrigued and highly motivated by the fledgling science of cosmology in the early 1970s, I first focused on finishing my graduate research to get my $\mathrm{Ph} . \mathrm{D}$. I did continue to pay attention to cosmology. One important factor was that Steven Weinberg was at MIT at the time giving a cosmology course whose notes eventually turned into his book, Gravitation and Cosmology. I was not able to attend all the lectures, but did get a lot of the notes and later the book. Weinberg's clear interest and seriousness added credibility to cosmology among my colleagues. This provided a foundation and piqued my interest in the field while I was spending most of my graduate student time doing particle physics.

My Ph.D. research involved testing a rule of weak force decays that the change in charge of a kaon in the decay was equal to the change in strangeness. For this research, four graduate students: Orrin Fackler, Jim Martin, Lauren Sompayrac, and myself, under the direction of my advisor Professor David Frisch (MIT Physics Department), used a special beam of $K^{+}$into a compact platinum target in the front of a magnetic spectrometer to produce $K^{0}$ 's and observe their decays in particle detectors inside the magnetic field. This was a highly technical and exacting experiment. We (Fackler et al., 1973; Smoot et al., 1975) found that the $\Delta S=\Delta Q$ rule (change in strangeness is matched by the change in charge of the particle decaying) was followed in the weak force decays. This rule is now understood as an automatic consequence of the quark model. This effort illustrates the temporal progress of science and how new young students are trained to do science. In our case, Professor Frisch gave us great and challenging tasks and responsibilities. He had us work independently much of the time, but there were people we could easily ask for advice and training. Now I was ready to move on and begin life as a newly minted postdoctoral scholar and find such a position. I investigated and interviewed for a number of jobs. Most of these were in particle physics which 
matched my training and my advisor's contacts. However, one interview was with Professor Luis Alvarez's group at Berkeley and, in particular, with a section that had been involved in trying to use energetic cosmic rays to push the frontiers of particle physics. They had met with a ballooning disaster in the program, were slowed in the original goals, and were looking to move in a new area. They were interested in flying a superconducting magnetic spectrometer to investigate the cosmic rays. Alvarez, like nearly all particle physicists at that time, knew that in every high energy interaction the conversion of energy into matter involved the production of equal amount of antimatter. Berkeley, in particular the Lawrence Berkeley National Laboratory, had been the scene for the discovery of antiproton and antineutron which established in everyone's mind that for every particle there was a matching antiparticle. Classically trained particle physicists thought at that time that in the big bang model there would be equal amounts of matter and antimatter. The question was then, "Where was the antimatter?" We had a good idea that there was none on earth and probably not in the solar system or we would be witnessing annihilation of matter and antimatter. Hannes Alfven, an acquaintance of Alvarez, had a cosmological model in which there was an annihilation Leiden-frost barrier that kept most of the matter and antimatter regions separate on a moderately large scale. Alfven encouraged Alvarez and the group to search for some leakage between the regions in the most likely sample of material from great distances, the cosmic rays. A cosmic ray magnetic spectrometer was an ideal instrument for this antimatter search. The skills and techniques I had learned as a graduate student matched well with those needed for this research and Luis Alvarez and his colleagues, specifically Larry Smith, Mike Wahlig, and Andrew Buffington, recruited and encouraged me to join them in this effort.

We, including a number of very able technicians and engineers, designed, built, and flew, a number of times, superconducting magnetic spectrometers observing a sample of cosmic rays. As our search progressed our limits got progressively lower down to one in a thousand or less and then one in ten thousand or less. The first limit gets one out past the near neighborhood of stars. The second takes one to our whole galaxy, and perhaps beyond, with evidence that there was little or any antimatter compared to the matter on that scale. To me the question changed from "Where is the antimatter?" to "Why is there an excess of matter over antimatter in our universe?" This currently remains one of the major questions of cosmology. Bear in mind that we have strong reasons to believe that there was an equal amount of matter and antimatter in the very early universe. At early times the cosmic background radiation photons had enough energy to produce particleantiparticle pairs and a simple thermal equilibrium would have essentially the same number of each species of particle and corresponding antiparticle as photons in the very early universe. Currently there are more than a billion CMB photons for every proton and neutron (and thus every electron). In the very early universe there would have been essentially the same number (per degree of freedom weighting) of every particle and antiparticle and all would have been relativistic behaving very much like photons or neutrinos all in strong thermal equilibrium. As the universe expanded and cooled, eventually the particles and antiparticles annihilated into lighter things including the CBR photons which by then were too cool to drive the reaction back the other way. Without some imbalance developing, there would both be much less matter around in the present and still be equal amounts of matter and antimatter separated in their sparseness.

In 1964, updated and clarified through 1986 as the need grew, Andrei D. Sakharov put forth the necessary conditions for what he called the baryon asymmetry (matter over antimatter excess) to exist: (i) baryon number violation, (ii) $C P$ violation, and (iii) nonequilibrium. Since that time theorists have been trying to find the correct theory and experimentalists evidence for these conditions.

During the later phases of these antimatter-search observations, I began to consider what to do next. Should we make an improved version of the experiment and probe deeper or should I strike off on something new? Alvarez offered the advice that one should periodically review what new developments had taken place. I distilled and codified his advice and other experience into: When you reach a natural pause, check as to see what new avenues are open because of (i) new scientific knowledge and ideas, (ii) new instrumentation and techniques that open new areas for research, and (iii) new facilities, infrastructure, or other support. An important ingredient was: What things could be brought together to enable significant research progress? A lot of judgment is necessary in this process.

In 1973 I dug out the 1967 paper by Dennis Sciama pointing out that one could test Mach's principle and measure the rotation of the universe by the effect that rotation would have on the cosmic microwave background. It was not that specific on what the anisotropy pattern should be. There was also a 1969 paper by Stephen Hawking (1969) which did provide cases for many Bianchi models, but was difficult slogging for the nonexpert. Unfortunately, there was no clear idea of how fast the universe should be rotating except by analogy with the rotation of every thing in the universe from electrons to galaxies. This was insufficient to convince my colleagues or others that this was a measurement worth pursuing.

In 1971 Jim Peebles published his book Physical Cosmology which was much more astrophysically and observationally oriented than Steven Weinberg's Gravitation and Cosmology. In Physical Cosmology Peebles had a section called "Applications of the primeval fireball." In this section Peebles had a well developed discussion of the topic of what were the implications of the "possibly discovered primeval fireball," i.e., the cosmic microwave background. Peebles' writing was clear and easy to understand by nonspecialists. One application that Peebles 
laid out was entitled "The aether drift experiment" in which one could use the CMB (zero net momentum of the radiation frame) as a reference to measure one's motion relative to the natural frame to describe the big bang expansion of the universe. The predicted temperature variation with angle $\theta$ to direction of motion due to the Doppler effect produced by the observer's motion is

$$
T(\theta)=T_{0} \gamma /(1-\vec{\beta} \cdot \hat{n}) \simeq T_{0}(1+\beta \cos \theta),
$$

where $\vec{\beta}=\vec{v} / c$ and $\hat{n}$ is the direction of observation. Here was a well-defined project with an easy to calculate minimum signal. Astronomers knew that the solar system was moving as it orbited along with the rotation of our galaxy. The orbital speed is known to be about $200 \mathrm{~km} / \mathrm{s}$ or about $v / c=\beta=0.7 \times 10^{-3}$. This gives an expected signal of about $2 \mathrm{mK}(0.002 \mathrm{~K})$. Astronomers who thought about it also thought our galaxy and Andromeda were co-orbiting each other so that there was an additional component of motion. But very, very few even thought about it at the time. There were a couple of papers with predictions.

The first was Dennis W. Sciama's 1967 paper "Peculiar velocity of the sun and the cosmic microwave background" which predicted "The sun's peculiar velocity with respect to distant galaxies is roughly estimated from the red-shift data for nearby galaxies to be $\sim 400 \mathrm{~km} / \mathrm{sec}$ toward $l^{I I} \sim 335^{\circ}, b^{I I} \sim 7^{\circ}$. Future observations on the angular distribution of the cosmic microwave background should be able to test this estimate, if the background has a cosmological origin. If the test is successful it would imply that a "local" inertial frame is nonrotating with respect to distant matter to an accuracy of $10^{-3} \mathrm{sec}$ of arc per century, which would represent a 5000-fold increase of accuracy." The second paper was a follow up of the first by J. M. Stewart and D. W. Sciama (1967) entitled "Peculiar velocity of the sun and its relation to the cosmic microwave background." Its abstract summarized "If the microwave blackbody radiation is both cosmological and isotropic, it will only be isotropic to an observer who is at rest in the rest frame of distant matter which last scattered the radiation. In this article an estimate is made of the velocity of the sun relative to distant matter, from which a prediction can be made of the anisotropy to be expected in the microwave radiation. It will soon be possible to compare this prediction with experimental results."

\section{Why not seek the seeds of galaxy formation first?}

Why not seek the seeds of galaxy formation which at the time was predicted to be at the same level? The angular scales of the anticipated signals were very different. One of the largest (angular-size) clusters on the sky was the Coma cluster which is approximately half a degree on the sky. Most clusters are in the arcminute range and galaxies are in the arcsecond range. With the receiver technology of the time, observations would have to be made at long wavelengths and that would require very large radiotelescopes dedicated for long periods of time. The radiotelescopes were not designed for this type of observation and thus prone to a number of potential systematic effects including significant ground signal pickup. One could readily estimate the expected angular scales for what was then thought to be a universe full mostly of isolated galaxies in some Poisson distributed fashion. One could estimate the causal horizon to be of order $2^{\circ}$ and primordial galaxy seeds as one hundredth that angular size (roughly an arcminute or so). In this old picture one would expect a sky speckled with tiny arcminute spots at the $\mathrm{mK}$ level, while the Doppler effect from the aether drift, nonuniform Hubble expansion, or the rotation of the universe promised signals that were large features and coherent on the sky that might unveil new physics (see Fig. 2).

\section{E. Beginning the new aether drift experiment}

So now here was a project that had a guaranteed signal of well-defined angular dependence, and amplitude. This made it a good candidate to propose to colleagues, funding agencies, etc. One problem to overcome was the strong prejudice of good scientists who learned the lesson of the Michelson and Morley experiment and special relativity that there were no preferred frames of reference. There was an education job to convince them that this did not violate special relativity but did find a frame in which the expansion of the universe looked particularly simple. More modern efforts to find violations of special relativity look to this reference frame as the natural frame that would be special so that perhaps the suspicions were not fully unfounded. We had to change the name to "the new aether drift experiment" and present careful arguments as the title "aether drift experiment" was too reminiscent of the Michelson and morley ether drift experiment.

With that behind us my colleagues Rich Muller and Terry Mast were interested enough to learn more and begin outlining the experiment and encouraging and winning over others. Eventually, enough colleagues were convinced that some of the skilled technical staff in the group could be used to help develop the experiment. Key technical people were Jon Aymon, software; Hal Dougherty, mechanical; John Gibson, electronics, Robbie Smits, rotation system; John Yamada, technical assembly. Seed funding for components and shops was first procured and then proposals to NASA and so forth as the experiment began to form. A key step was recruiting graduate student Marc V. Gorenstein to work on the project. I had known Marc as an undergraduate at MIT before he came to Berkeley Physics graduate school and this connection helped just as Mike Wahlig and Andy Buffington had been at MIT in Frisch's group while I was an undergraduate and then new graduate student before they had come to Berkeley. There was a chain of contacts, familiarity, and confidence that helped make connections.

Now we had a nucleus of a team and a well-defined objective: build an instrument with sufficient sensitivity and precision to measure a CMB anisotropy at the $2 \mathrm{mK}$ 


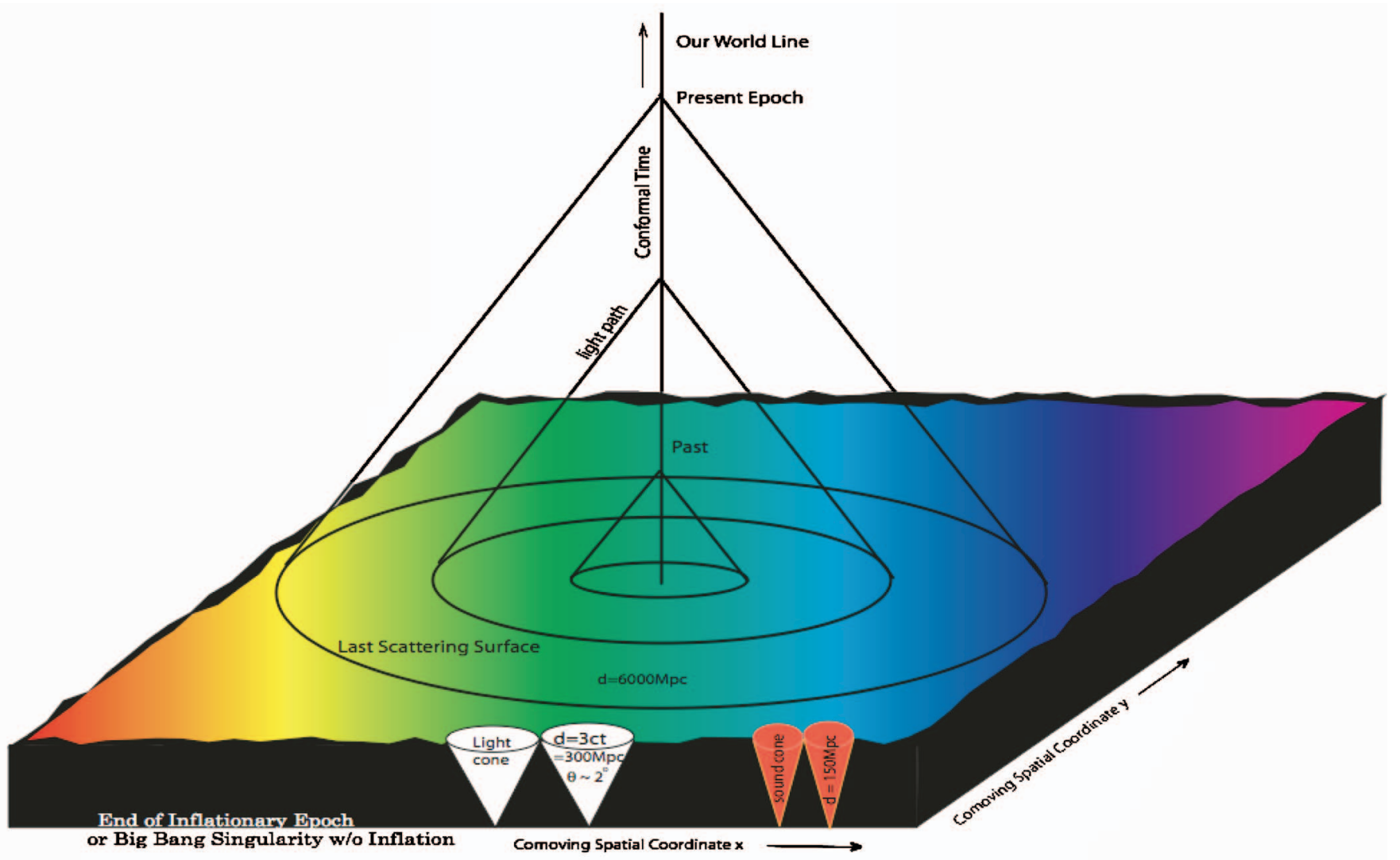

FIG. 2. (Color) A space-time diagram in units of conformal time (vertical) $\eta=\int c d t / a(t)$, where $a(t)$ is the scale factor of the universe, and comoving coordinates which are converted to physical distance by multiplying by the scale factor $a(t)$. In these coordinates light travels on a $45^{\circ}$ angle. The line in the center is our matter's path through time shown with no peculiar motion (very small in practice). The universe is shown opaque until the last scattering surface from either the end of the inflationary epoch or the big bang singularity. The thickness of this is shown exaggerated relative to the subsequent elapsed time until the present (now) so as to show the causal horizon (distance that could be covered by the speed of light $d=3 c t$ in physical units) and the sound horizon (distance that would be covered by speed of sound in the early universe). These two horizons have special imprint upon the physical structures in the universe.

$\left(10^{-3}\right)$ level on large angular scales. We began work and, with previous unfortunate experience with scientific ballooning, were considering using a differential microwave radiometer (DMR) on a U2 aircraft. Terry Mast peeled away to work on the nascent $10-\mathrm{m}$ telescope project. We were luckily joined by Tony Tyson taking a sabbatical to Berkeley from Bell Labs where he was working on developing gravity wave detectors. Tony had become expert on low noise detectors and vibration isolation, two key technologies we would need in this endeavor and which were important in the development of the DMR.

\section{F. Context}

1973 was when I began moving into work on CMB but it was a field that was already active on the east coast in significant part due to the activities of Jim Peebles and Bob Dicke leading to pioneering work by David Wilkinson, first with P. Roll in 1965 and then a succession of graduate students, e.g., beginning with Bruce Partridge in 1967. There was spinoff from Princeton to MIT of Rainer Weiss who worked with Dirk Muehlner there. Both of these groups began with observations of the
CMB spectrum and branched to anisotropy measurements. I chose to begin with anisotropy and move to the spectrum and other aspects later.

In 1970 Joe Silk came to Berkeley and began the theoretical cosmology effort creating a west coast effort and began to influence his colleagues to consider cosmological observations. Soon afterwards Professor Paul Richards began a program taking on graduate students John Mather and then Dave Woody. Richards' program develops bolometers and Michelson interferometer for spectrum observations and these are the precursor for COBE FIRAS. Significantly later these bolometers' descendants become a key detector for CMB anisotropy observations. See the proceedings by my co-recipient John Mather.

Joe Silk and I developed a symbiotic student training program. Those that he wanted to get involved in analysis and understanding of observations would apprentice with me for a semester or a year. These students then helped with defining possible observations or working out some theory needed. Some of the students involved in this over the years were Mike Wilson, John Negroponte, and Eric Gawiser. 
G. Why did we need such a strong team and effort?

The anticipated signal was at the level of one thousandth of the CMB $(\sim 3 \mathrm{~K})$ which in turn was one hundredth of the ambient temperature $\sim 300 \mathrm{~K}$. The equivalent radio-signal receiver noises were in the same range. Thus the anisotropy was anticipated to be at a part in one hundred thousand $\left(10^{-5}\right)$ of the noisy backgrounds. To have a significant measurement we would need to probe down to one tenth that level or a part in a million $\left(10^{-6}\right)$. Thus we needed sensitivity to low signal levels which meant relatively long observation and stability of the instrument.

What were the techniques we could use? First we could use a technique championed by Bob Dicke in the 1940s that rapidly switches the receiver input between two sources and looks at the difference. The more the comparison was done with signals at the same level and the more quickly the inputs were switched, the less important would be the inevitable instrumental drifts due to the intrinsic $1 / f$ electronic device noise and the roughly $1 / f^{2}$ thermal environmental fluctuations that would prevent direct measurements of the CMB to the part in a million level. For measurements of the CMB one needed a reference at or near its $3 \mathrm{~K}$ temperature. For spectrum measurement one would use a reference load cooled with liquid helium to achieve this. Our approach for the anisotropy experiment was to use two identical antennas pointing at different portions of the sky and switch rapidly between them. This configuration we called a differential microwave radiometer (DMR).

It was then necessary to exclude, reject, average out other signals and sources of noise. We had to choose an observation frequency in which the CMB fluctuations would be larger than (or at least distinguishable from) those from other sources, particularly our own galaxy. This led us to choose a roughly $1 \mathrm{~cm}$ wavelength and to choose where we looked in the sky. Except near the galactic plane the CMB anisotropy should dominate and $1 \mathrm{~cm}$ was a wavelength that was relatively minimal atmospheric emission and so had been chosen by microwave pioneers as $K$ band. When it was realized that $K$ band had a water line in it, the band had been readjusted by microwave engineers to be $K_{A}$ band. Thus there were standard microwave components that were optimized for this wavelength range.

The electrical noise of the receiver produced background fluctuations that were of order

$$
\begin{aligned}
\delta T_{\text {rms }}=\frac{2 T_{\text {system }}}{\sqrt{B \tau}}+\frac{\Delta G}{G} T_{\text {diff }} & \sim \frac{27 \mathrm{mK}}{\sqrt{\tau / \mathrm{sec}}}+100 \mathrm{mK} \frac{\Delta G}{G} \\
& \sim \frac{0.5 \mathrm{mK}}{\sqrt{\tau / \mathrm{h}}}
\end{aligned}
$$

where $T_{\text {system }} \sim 300 \mathrm{~K}$ was the effective receiver noise temperature of ambient temperature receivers of that epoch, $B$ was the bandwidth on the order of $500 \mathrm{MHz}$, and $\tau$ was the observation time, and $\Delta G$ was the change in receiver power gain $G$ in the time period of the observations preferably set by the switching between inputs of receiver whose effective temperature difference was $T_{\text {diff }}$. (Note that these two effects should in general be added in quadrature as they would be uncorrelated.) The first is simply due to the variance of $\bar{n}^{2}+\bar{n}$ in the number of photons observed due to thermal fluctuations of blackbody radiation and the second to receiver gain drift. By making the temperature difference $T_{\text {diff }}$ small, $\left|T_{\text {diff }}\right|<0.1 \mathrm{~K}$ was possible to achieve, one could hope not to increase the rms noise significantly as long as the receiver gain variation was kept significantly less than $0.5 \%$ for the switching time for the required sensitivity of about $0.3 \mathrm{mK}$. To achieve this level of sensitivity we would need to observe each patch of the sky for about two hours.

Thus our plan was to average down the random noise in two hour chunks but we also had to exclude signals that were not random. A key issue was the rejection of signals coming from off the main beam axis. A fundamental property of optics is that diffraction will cause the beam to have off-axis response. The usual antenna technology of the time with the lowest sidelobes (offaxis response) was the "standard gain horn" which has the optimum gain for a simple pyramidal horn configuration. This horn basically is a smoothly expanding waveguide and has in one plane ( $E$ plane which is in the same plane as the electric field vector) a uniform illumination to the edge of the horn. The illumination in the orthogonal direction ( $H$ plane) varies as a sine wave with zero amplitude at the waveguide (horn) edges and peaking in the middle. This field configuration is simply the lowest and best supported mode of the waveguide and the one for which all the other components are designed to utilize.

The far field (equivalent to the beam response) is simply the Fourier transform of the aperture electric field. The Fourier transform of an electric field that is zero outside the horn and uniform inside the horn is the familiar to physicists' $\sin (x) / x$ pattern. For a reasonable horn size the beam is fairly broad, but also, more importantly, the side lobes are typically only down by a factor of 10000 at $90^{\circ}$ to the beam axis. Since the ground is nonuniform and a million times greater than the anticipated anisotropy signal level, we needed a better solution. I decided that I had to learn antenna theory and find what could be done to get to lower sidelobes. The $H$-plane pattern with its sine wave illumination, specifically the tapering of the field to zero at the edges of the horn aperture, has quite low side lobes and points the path towards the solution. One would want electric field illumination that tapered smoothly to zero at the aperture edges. Ideally one would like the field and its derivative to be zero at the edge, even though that meant that for the given aperture diameter the forward gain was lower since it was under illuminated relative to uni- 
form illumination (hence the optimum standard gain horn design). The fact that the $H$-plane beam pattern was quite low meant that one could achieve the necessary low off-axis response as long as the electric field tapered reasonably to zero.

Another way to look at the issue is that the energy in the wave is stored in the electric field and considering a wave going in the time-reversed direction, the issue was how to take the field tightly coupled to the waveguide and send it out the antenna and have it separate from the antenna and match into propagating freely in space. If the electric field is not zero at the metal on the end of the horn near the aperture, then the electric field generates currents in the metal to make the field close to zero in the conducting metal. These currents then cause field to propagate out at other directions. So again by the end of the antenna we need the electric field decoupled and zero at the metal surface. There are two approaches to this that eventually were used in the two CMB instruments on the COBE satellite. The first approach is to flare the ends of the horns very much like the bell on a trumpet or a trombone which as musical instruments have a similar issue of emitting sound waves from tightly coupled at the mouth piece but freely propagating once they leave the horn. Hence the pictures I showed of the Princeton (Wilkinson group) anisotropy experiment with the musical instrument bells on the end of their hornsbut for receiving electromagnetic (EM) waves, not transmitting them. The electromagnetic wave prefers to propagate along a straight path and effectively peels away successively along the curve. This approach has the benefit of working for a large range (bandwidth) of frequencies and the disadvantage of extending the size of the aperture substantially - the more one needs off-axis rejection the larger the flare must be. This was the approach used in the COBE FIRAS instrument where there was a single large external horn antenna that had to work well over an extended wavelength range.

The second approach which I eventually pursued was to separate the electric field from the antenna very early and use the rest of the antenna to keep defining and shaping the beam and then to put in quarter wavelength deep grooves at the ends of the aperture. Quarter wavelength deep grooves would then force currents exactly out of phase with the electric field (1/4 wavelength down and $1 / 4$ wavelength back meant $1 / 2$ wavelength or $180^{\circ}$ out of phase). This chokes off the surface currents in the horn aperture and does not allow them to go out and around the horn to make far and back lobes. The issue at the horn throat is to excite a second mode that has the property that at the center of the beam its field is in phase with the standard mode but at the $E$-plane edges its field is out of phase and just cancels the electric field from the standard first mode giving a field pattern that is very similar to the $H$ plane and has very low side lobes. I studied the literature, consulted with engineers from TRG Alpha in Boston, Massachusetts, and got from Jet Propulsion Laboratory (JPL) a copy of their software JPLHORN for calculating beam patterns which I modified and used. Soon it was clear that one could do this quite well with what is called a corrugated-horn antenna, especially in the case of a conical horn. The first groove needed to be a half wavelength deep so as to not develop too much reflection and then one could either tune and go directly to quarter wavelength (in the cone) deep grooves which was easier to fabricate or, as we did on later horns, taper the groove depth from half wavelength to quarter wavelength depth in a few (five to ten) grooves and have the remaining thirty or so grooves at quarter wavelength depth. This configuration produced very low far side lobes, in a very compact configuration, and had very low losses in the antenna since the electric field did not produce significant currents in the antenna wall. It had the draw back that it was relatively expensive to make since it required a very good machinist working on fairly large forged aluminum blocks to cut in all those grooves precisely. This development was sufficiently successful that eventually we had to develop new techniques to observe side lobes this low (necessary for the COBE DMR) and used an antenna range at JPL sited on the edge of a mesa (Janssen et al., 1979). This work was repeated for the COBE DMR antennas at Goddard Space Flight Center (GSFC) in a specially developed range (Toral et al., 1989). This was a key development since to measure the CMB anisotropy precisely, one must achieve off-axis rejection to a part in a billion level or better, and for the DMRs the antennas needed to be sufficiently compact to fit within the available space.

The design called for two identical horns whose output was rapidly and alternately switched into the receiver. As long as the horns were identical and they looked out through identical atmosphere the measurement should be sufficient. However, things are not perfectly identical and so we had to have a back up which was that we must rotate the receiver and interchange the position of the antennas on the sky so we could separate out any intrinsic signal from the instrument from that coming from the sky. This was a generic issue which over the years my students referred to as Smoot's switch rule: As soon as one introduced a switching (or technique) to cancel out or correct for an effect, one had two new effects to be concerned with: (i) did the device produce a signal itself and (ii) did the process (e.g., rotating the instrument) produce a signal? These effects always occur at some level so one has to make sure that they are small and compensated for in the design. For example, the switching of the receiver input from one antenna output to another always introduces a spike, step, or some form of extra signal during the process, so one does not include that as part of the signal stream that continues on. Likewise the switch has a slight offset when connected to one antenna compared to the other. So one measures and adjusts this as well as possible and then makes sure to rotate the apparatus so that the sky signal is interchanged (and thus of opposite sign) as to which horn antenna it enters. Then one must check that the process of rotation does not change the state or performance of the DMR, e.g., from the earth's magnetic field, or other effects. In general, since we are measuring such a small 
signal, one had to be concerned to roughly third order in things as well as conduct many tests and analyses.

\section{H. The DMR and U2 observations}

Finally we had developed the instrumentation and approach to observe the $\mathrm{CMB}$ and to detect the first order anisotropy due to the motion of the instrument relative to the last scattering surface and thus the zero momentum frame for the CMB. The instrument used in the experiment was a differential microwave radiometer (DMR), eventually described in detail (Gorenstein et al., 1978). The vehicle of choice was the high-flying and very stable U2 jet aircraft famed for making flights from Turkey to Scandinavia as well as over other hot spots where high resolution (thus stable platform) photographs taken from high altitude were of use. The U2 had been converted to performing environmental and earth resources observations in a program run by the NASA Ames Center from Moffett Field, California. After a series of flights and subsequent data processing and analysis we detected (Smoot et al., 1977) this first order, dipole anisotropy, as well as showing that it was the dominant large scale signal on the sky (Gorenstein and Smoot, 1981). Later we were to put together a program to make these observations from Peru so that we could see that the pattern held over the southern sky as well (Smoot and Lubin, 1979). The U2 experiment revealed that the temperature varied smoothly from $-3.5 \mathrm{mK}$ in a direction near the constellation Aquarius to $+3.5 \mathrm{mK}$ in the direction near the constellation Leo (see Fig. 3). Surprisingly, this meant that our solar system was moving at $350 \mathrm{~km} / \mathrm{s}$, nearly opposite to the direction that was expected from the rotation around the galactic center. This result forced us to conclude that the Milky Way was moving at a speed of about $600 \mathrm{~km} / \mathrm{s}$ in the direction near the constellation Leo. This motion was not expected in the model where galaxies are simply following undisturbed world lines given by simple Hubble expansion of the universe, which was the idealized version that most cosmic astronomers held to at the time. It also implied that the Andromeda Galaxy as well as the many smaller members of the local group were also moving along with similar velocities. This group motion implied that there is a gravitational center (later named the "great attractor") of a huge clump of matter relatively far away so that its pull was uniform enough not to disrupt the weakly bound local group (see Fig. 4).

Even though the dipole was not of direct cosmological origin, it carried a significant meaning on how matter is organized in the universe and therefore on what conditions must exist at the onset of the big bang. Once we could convince astronomers that this was correct and the great attractor or equivalent could be found, then we would also achieve Sciama's test of Mach's principle and our relative rotation with respect to the distant matter in the universe. It took some time before astronomers took this seriously (some encouragement from UCLA astronomer George Abell helped) and work began that eventually led to understanding that the existence of clusters and superclusters of galaxies and our motion as well as other bulk motions were a natural consequence of the large scale organization of matter. At the same time searches on larger and larger scales (shells at greater radius and redshift) began to converge on the "right" answer given by the CMB. The current best observed dipole $(3.358 \pm 0.017 \mathrm{mK})$ indicates that the solar system is moving at $368 \pm 2 \mathrm{~km} / \mathrm{sec}$ relative to the observable universe in the direction galactic longitude $l$ $=263.86^{\circ}$ and latitude $b=48.25^{\circ}$ with an uncertainty slightly smaller than $0.1^{\circ}$ (Jarosik et al., 2006). This is quite far from the galactic rotation direction (nominally $250 \mathrm{~km} / \mathrm{s}$ toward $l=90^{\circ}$ and $b=0^{\circ}$ ).

Surprisingly, it is closer to the original prediction in his 1967 papers by Sciama of $\sim 400 \mathrm{~km} / \mathrm{sec}$ toward $l^{\mathrm{II}}$ $\sim 335^{\circ}, b^{\mathrm{II}} \sim 7^{\circ}$, which was based upon very sketchy observations of the time. The meaning we could take from this was that stepping back and taking a skeptical look with a clear mind did allow one to realize that there was large scale structure in the universe and some expected variation from simple Hubble flow due to the small acceleration of distant gravitational attraction operating over billions of years. If large voids and superclusters could form, then there must be some bulk flows. The motion of our galaxy is just a bit above the norm; hence the need for the great attractor which is one of many in the universe.

Detection of the intrinsic CMB anisotropy was a great technical challenge in its own right, for it required an accuracy of one part in 100000 . Galactic and extragalactic emissions, foreground emissions, and noise from the instruments themselves added noise to the measurements which were much larger than the CMB signal. Even U2 flight experiments, which were carried out at altitudes above $65000 \mathrm{ft}(20 \mathrm{~km})$, and which were above $95 \%$ of atmosphere (balloon experiments are also performed at similar condition), were not enough to catch such a subtle primordial whisper. Space-based observations would provide far better results, so the next phase of the $\mathrm{CMB}$ measurements moved on to satellite-borne experiments.

\section{Polarization of the CMB}

Penzias and Wilson set the first limit on the polarization of the CMB at less than $10 \%$. Then Martin Rees came forward with his prediction (Rees, 1968) that the CMB should be linearly polarized at what should be a few percent of any intrinsic anisotropy. In the 1970s then graduate student George Nanos in Wilkinson's Princeton group (Nanos, 1979) and our group (Lubin and Smoot, 1979; Lubin et al., 1983) began observations to improve the limits on the polarization of the CMB and test Rees's prediction. At the time we were still thinking in terms of classical large scale anisotropy. The simplest version would be the case if the universe was expanding at different rates in the three orthogonal directions. The optimal case would be a quadrupolar axisymmetric ex- 

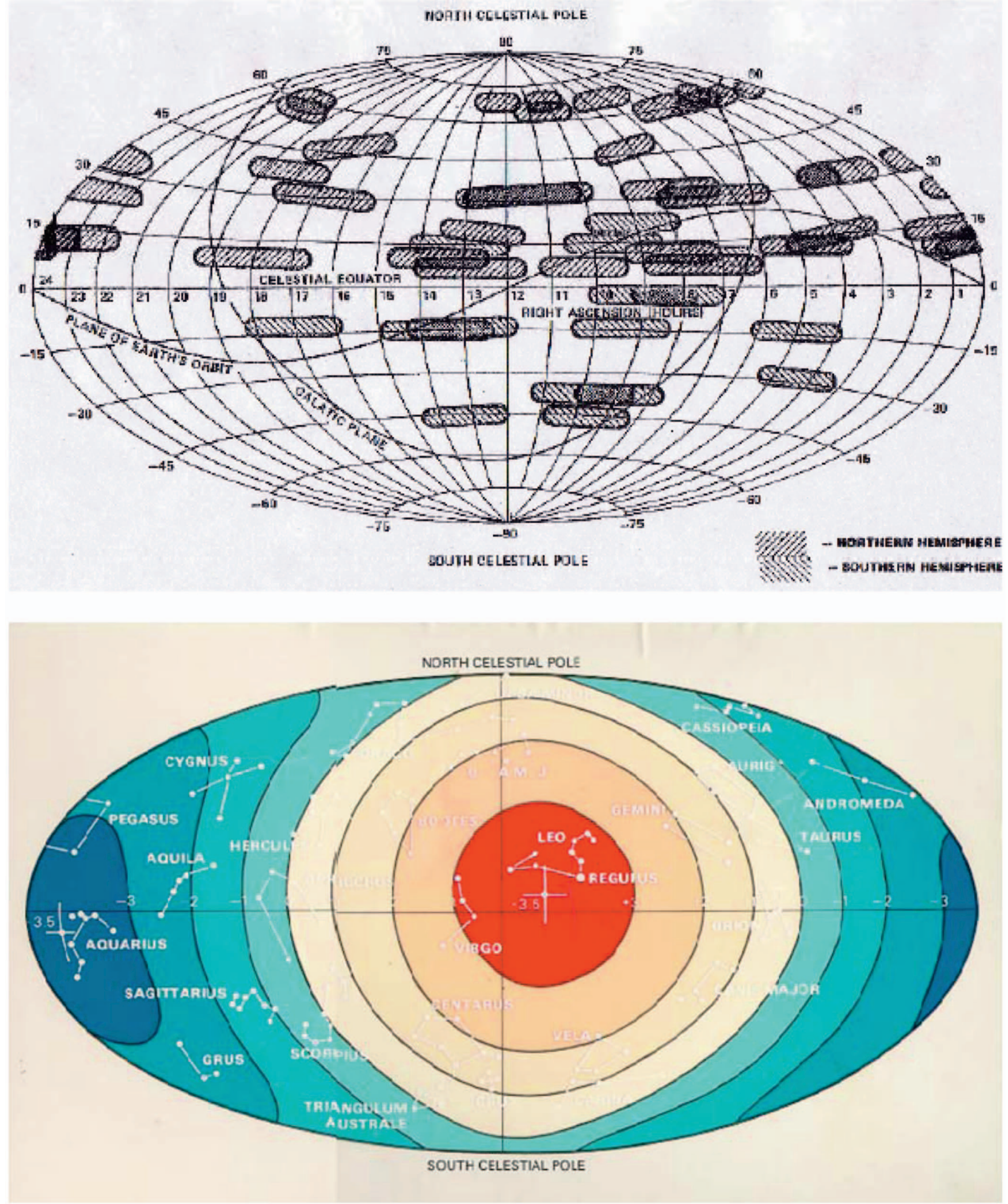

FIG. 3. (Color) Dipole anisotropy measured by U2 flight experiment (1976). Top panel: Sky covered by U2 flight experiments in northern and southern hemispheres. Bottom panel: The dipole map made by U2 experiments. The red spot centered near the constellation Leo indicates $+3.5 \mathrm{mK}$ from the median background temperature and the blue spot centered near the Aquarius is $-3.5 \mathrm{mK}$ region (Smoot et al., 1977; Gorenstein et al., 1978). 


\section{VELOCITY COMPONENTS OF THE OBSERVED CMB DIPOLE}

COBE AROUND EARTH

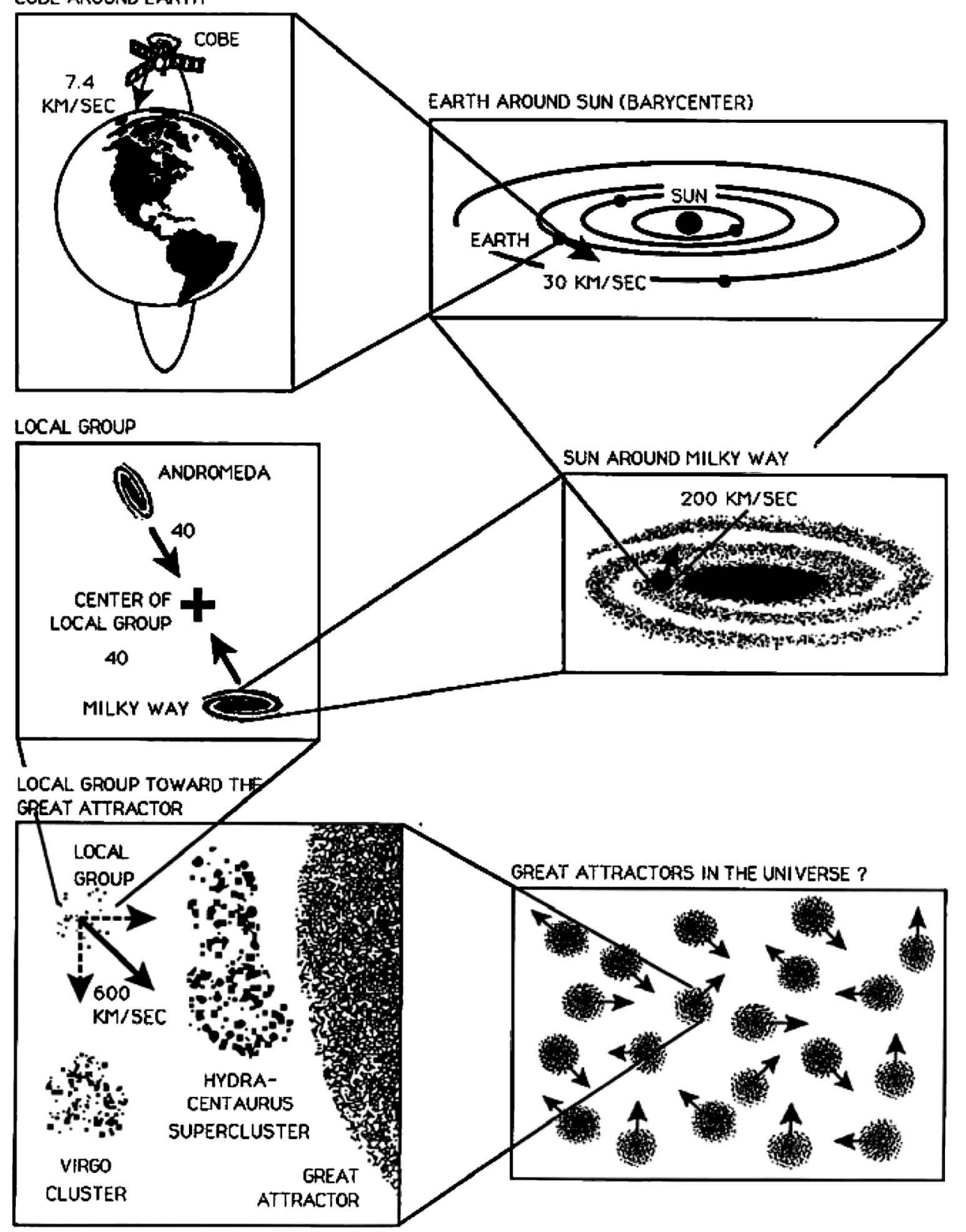

FIG. 4. Dipole anisotropy results from the sum of many components of velocity due to gravitational attraction of various mass concentrations.

pansion which would couple maximally to linear polarization.

Nanos completed his thesis observations and work and published in 1979. Nanos's abstract reads "An attempt is made to detect linear polarization in the 2.7 background at a wavelength of $3.2 \mathrm{~cm}$, using a Faradayswitched polarimeter pointed to the zenith where the earth's rotation carries through a circle of constant (40.35 deg N) declination. A two-step calibration process was employed. First, the change in dc voltage at the second detector was measured with a $300 \mathrm{~K}$ absorber to determine the gain of the microwave front end. Then, by inserting a known ac voltage at the lock-in frequency at the same point, the rest of the receiver was calibrated." The null result was interpreted using Rees' axially symmetric model as an upper limit on asymmetry in the Hubble expansion. Nanos then went onto a career in the Navy becoming Vice Admiral and later director of Los Alamos National Laboratory.

Once the U2 experiment was progressing well and we had developed techniques and instrumentation, I felt it was time to begin polarization observations. I recruited graduate student Phil Lubin and convinced him to do, for his $\mathrm{Ph} . \mathrm{D}$. thesis, a polarization experiment at $1 \mathrm{~cm}$ 
wavelength. We calculated that observations could be made from the ground using the basic instrumentation that we developed for the U2 DMR but with a single antenna that accepted both linear polarizations and a switch to chop the receiver input quickly between the two polarizations. After study we determined that the atmosphere was sufficiently unpolarized that we could make our observations from the ground. We needed only point the antenna up and the rotation of the earth would sweep out a strip on the sky. Once that worked we could tip the radiometer slightly north or south and obtain strips on the sky. Hal Dougherty produced the mechanical system construction and John Gibson produced the electronics and power supplies. We had to design the system to rotate about its axis to separate instrumental effects from sky signals. Phil then tuned the system up and began observations and ultimately found it worked well and we gathered data mostly from Berkeley but also some from the southern hemisphere (Lima, Peru).

Later, with improved design and construction by our outstanding mechanical tech, Hal Dougherty, we extended to $3 \mathrm{~cm}$ wavelength for Philip Melese d'Hospital's project and then on to $0.3 \mathrm{~cm}$ wavelength. Our data were continuing measurements of the linear polarization of the cosmic background radiation as well as provided the first measurement of the circular polarization. We surveyed 11 declinations for linear polarization and one declination for circular polarization, all at $9 \mathrm{~mm}$ wavelength (Lubin and Smoot, 1979; Lubin et al., 1983). We found no evidence for either a significant linear or circular component with statistical errors on the linear component of $20-60 \mu \mathrm{K}$ for various models. Our linear polarization, a $95 \%$ confidence level limit of $0.1 \mathrm{mK}(0.00003)$ for an axisymmetric anisotropic model was achieved, while for spherical harmonics through third order, a corresponding limit of $0.2 \mathrm{mK}$ was achieved. For a declination of $37^{\circ}$, a limit of $12 \mathrm{mK}$ was placed on the time-varying component and $20 \mathrm{mK}$ on the dc component of the circular polarization at the $95 \%$ confidence level. At $37^{\circ}$ declination, the sensitivity per beam patch $\left(7^{\circ}\right)$ was $0.2 \mathrm{mK}$.

After these observations, interest in the $\mathrm{CMB}$ polarization died down significantly until the COBE DMR discovery of intrinsic $\mathrm{CMB}$ anisotropies and since then investigations of the $\mathrm{CMB}$ polarization has become a major interest of the field.

\section{J. Balloon-borne anisotropy at $3 \mathrm{~mm}$ wavelength}

After the detection of the dipole anisotropy and first estimated map of the CMB anisotropy, it was time to develop instruments to make a real map. Phil Lubin, now a new Ph.D., and I conceived of a new detector with much greater sensitivity than the $\mathrm{U} 2$ receiver so as to be able to make a map with observations of reasonable time duration. We knew that to improve the sensitivity of the receiver it would need to be cooled to cryogenic temperatures. We obtained a liquid-helium dewar and $\mathrm{Hal}$ modified it to hold the antenna and the receiver front end and so forth. We needed to move to higher frequency to keep the system compact and then, as a result, needed to be higher in the atmosphere to avoid atmospheric fluctuations. This led us to a cryogenic, balloon-borne system design. It was then up to us to produce the front end of the receiver while $\mathrm{Hal}$ and John produced the mechanical systems and the electronics. A critical piece of the effort was developing a large mechanical chopper to switch quickly where the beam intersected the sky and a reliable pop-up calibrator. I also recruited a new graduate student, Gerald Epstein, for whom this was his Ph.D. research project. After two flights in the northern hemisphere, Gerald had enough results for his thesis but we wanted to continue map making to cover the southern hemisphere. We then recruited Brazilian graduate student, Thyrso Villela, which was natural for balloon flights from Brazil. We were able to produce a map covering about $70 \%$ of the sky which showed the dipole anisotropy well and showed clearly that the higher order (quadrupole moment) and above were significantly lower. Equally important, the project showed that we could put together a compact cryogenic system that was capable of more sensitive observations of the CMB. This provided the confidence and heritage needed to improve the COBE DMR receivers, in midCOBE DMR development. This was a key piece of evidence which was presented to the GSFC engineering management and ultimately to NASA Headquarters to convince them to allow the change to passively (radiatively) cooled COBE DMRs as the suborbital experiments and theory began to indicate that we were going to need every bit of sensitivity we could muster.

This balloon-borne project later morphed into the MAX, MAXIMA, BOOMERANG, and MAXIPOL experiments when a collaboration with Paul Richards' group and the developing bolometers technology made bolometer arrays a real possibility. Phil Lubin moved to be a Professor at UCSB and continue collaborating. Gerald Epstein moved to work in science policy beginning at OTA.

Thyrso Villela took a position as a researcher and professor at INPE (Brazilian Space Agency) in San Jose dos Campos.

\section{K. Spectrum of the CMB}

Observations of the spectrum of the CMB started with the discovery observation by Penzias and Wilson combined with the original speculation that this was the relic radiation from the big bang and would to first order be a blackbody (Planckian) spectrum. Later theoretical studies confirmed that to high order one expected that the relic radiation should have blackbody spectrum because of the high level of thermal equilibrium expected in the early universe.

A noninteracting Planckian distribution of temperature $T_{i}$ at redshift $z_{i}$ transforms with the universal expansion to another Planckian distribution at redshift $z_{r}$ with temperature $T_{r} /\left(1+z_{r}\right)=T_{i} /\left(1+z_{i}\right)$. Hence thermal equilibrium, once established (e.g., at the nucleosynthe- 
sis epoch), is preserved by the expansion, even during and after the photons decoupled from matter at early times $z \sim 1089$. Because there are about $10^{9}$ photons per nucleon, the transition from the ionized primordial plasma to neutral atoms at $z \sim 1089$ does not significantly alter the CBR spectrum (Peebles, 1993).

Shortly after the Penzias and Wilson discovery and initial estimate of the $\mathrm{CMB}$ temperature, there were a number of observations and determinations or estimations of the temperature at various wavelengths which were the beginnings of the effort to establish that the CMB spectrum was blackbody. Dave Wilkinson and Peter Roll $(1966,1967)$ were pioneers in radiometric observations beginning on the roof of Jadwin Hall at Princeton. Wilkinson and colleagues, first Stokes and Partridge (Stokes et al., 1967), continued with a set of long wavelength observations from the White Mountain Research Station in California. This is a high altitude site operated by the University of California and one that is a good site for CMB observations because of its high altitude (12 $000 \mathrm{ft}$ ), dryness, and reasonable accessibility (a road that is open for about half the year). As mentioned above, by 1974 Professor Paul Richards began a program taking on graduate students, John Mather and Dave Woody. Richards' program develops bolometers and Michelson interferometer for spectrum observations and these are the precursor for COBE FIRAS. The FIRAS instrument design came directly from the original White Mountain instrument, which was then morphed to be the Woody and Richards balloon-borne instrument. The FIRAS team, led by John Mather, studied the results and performance of the Woody and Richards instrument and experiment and designed FIRAS both to be as symmetric as possible and to operate at the same temperature as that from the sky input. Another key feature was the sky simulating blackbody which was carefully designed, crafted and tested to be a very good blackbody at a well-defined temperature (see Tables II-IV).

In the early 1980s, during the Woody and Richards experimental effort, my group started an international collaboration to measure the low frequency portion of the spectrum complementary to Woody and Richards high frequency observations. This collaboration included the University of Milano group headed by Giorgio Sironi, the Bologna group of Nazareno Mandolesi, University of Padua theorists Luigi Danese and Gianfranco DeZotti, and the Haverford group led by Bruce Partridge. We carefully developed special radiometers to measure the spectrum at wavelengths of $12,6,3,1$, and $0.3 \mathrm{~cm}$ (frequencies of $2.5,5,10,30$, and $90 \mathrm{GHz}$ ). We developed very large $(0.75 \mathrm{~m}$ diameter $)$ liquid-heliumcooled $(3.8 \mathrm{~K})$ reference loads so as to have a blackbody source near to the temperature of the sky and CMB (see Fig. 5). We had to develop techniques, model of galactic emission and where it was low, and most importantly models of the residual high altitude atmospheric signal. We not only modeled the atmosphere but also conducted zenith scans and the Haverford group operated a full time atmospheric monitor. We conducted a series of
TABLE II. Values of the CMB temperature at $\nu \leq 11 \mathrm{GHz}$.

\begin{tabular}{|c|c|c|c|}
\hline $\begin{array}{l}\nu \\
(\mathrm{GHz})\end{array}$ & $\begin{array}{c}\lambda \\
(\mathrm{cm})\end{array}$ & $\begin{array}{l}T_{\mathrm{CMB}}^{\mathrm{th}} \\
(\mathrm{K})\end{array}$ & Reference \\
\hline 0.408 & 73.5 & $3.7 \pm 1.2$ & $\begin{array}{c}\text { Howell and Shakeshaft, } \\
1967 \mathrm{~b}\end{array}$ \\
\hline 0.6 & 50 & $3.0 \pm 1.2$ & Sironi et al., 1990 \\
\hline 0.610 & 49.1 & $3.7 \pm 1.2$ & $\begin{array}{l}\text { Howell and Shakeshaft, } \\
\text { 1967a }\end{array}$ \\
\hline 0.635 & 47.2 & $3.0 \pm 0.5$ & Stankevich et al., 1970 \\
\hline 0.820 & 36.6 & $2.7 \pm 1.6$ & Sironi et al., 1991 \\
\hline 1 & 30 & $2.5 \pm 0.3$ & $\begin{array}{c}\text { Pelyushenko and Stankevich, } \\
1969\end{array}$ \\
\hline 1.4 & 21.3 & $2.11 \pm 0.38$ & Levin et al., 1988 \\
\hline 1.42 & 21.2 & $3.2 \pm 1.0$ & Penzias and Wilson, 1965 \\
\hline 1.43 & 21 & $2.65_{-0.30}^{+0.33}$ & Staggs et al., 1996a \\
\hline 1.44 & 20.9 & $2.5 \pm 0.3$ & $\begin{array}{c}\text { Pelyushenko and Stankevich, } \\
1969\end{array}$ \\
\hline 1.45 & 20.7 & $2.8 \pm 0.6$ & $\begin{array}{c}\text { Howell and Shakeshaft, } \\
1966\end{array}$ \\
\hline 1.47 & 20.4 & $2.27 \pm 0.19$ & Bensadoun et al., 1993 \\
\hline 2 & 15 & $2.5 \pm 0.3$ & $\begin{array}{c}\text { Pelyushenko and Stankevich, } \\
1969\end{array}$ \\
\hline 2 & 15 & $2.55 \pm 0.14$ & Bersanelli et al., 1994 \\
\hline 2.3 & 13.1 & $2.66 \pm 0.7$ & Otoshi and Stelzreid, 1975 \\
\hline 2.5 & 12 & $2.71 \pm 0.21$ & Sironi et al., 1991 \\
\hline 3.8 & 7.9 & $2.64 \pm 0.06$ & De Amici et al., 1991 \\
\hline 4.08 & 7.35 & $3.5 \pm 1.0$ & Penzias and Wilson, 1965 \\
\hline 4.75 & 6.3 & $2.70 \pm 0.07$ & Mandolesi et al., 1986 \\
\hline 7.5 & 4.0 & $2.60 \pm 0.07$ & Kogut et al., 1990 \\
\hline 7.5 & 4.0 & $2.64 \pm 0.06$ & Levin et al., 1992 \\
\hline 9.4 & 3.2 & $3.0 \pm 0.5$ & Roll and Wilkinson, 1966 \\
\hline 9.4 & 3.2 & $2.69_{-0.21}^{+0.16}$ & Stokes et al., 1967 \\
\hline 10 & 3.0 & $2.62 \pm 0.058$ & Kogut et al., 1988 \\
\hline 10 & 3.0 & $2.721 \pm 0.010$ & Fixsen et al., 2004 \\
\hline 10.7 & 2.8 & $2.730 \pm 0.014$ & Staggs et al., 1996b \\
\hline
\end{tabular}

campaigns in successive summers at White Mountain and then for two successive years at the South Pole which is also a high and very dry site with a very stable and relatively small atmospheric signal. We published a series of papers on the theory, observations, and interpretation leading to a much improved set of measurements at long wavelengths (Danese and de Zotti, 1977, 1982; Smoot et al., 1983a, 1983b, 1984, 1987, 1988; Mather et al., 1994). The few additional observations that have tried to improve on these measurements have been generally balloon-borne versions with the same basic concept but above much more of the atmospheric signal. Over time a large number of people were involved including G. F. Smoot, LBL/UCB, G. De Amici, UCB, S.D. Friedman, UCB, C. Witebsky, UCB, N. Mandolesi, Bologna, R. B. Partridge, Haverford, G. Sironi, Milano, L. Danese, Padua, G. De Zotti, Padua, Marco Bersanelli, Milano, Alan Kogut, UCB, Steve Levin, UCB, Marc Bensadoun, UCB, S. Cortiglioni, Bologna, 
TABLE III. Values of the CMB temperature at frequencies $\nu>11 \mathrm{GHz}$. The measures from FIRAS, COBRA, and CN molecules are excluded.

\begin{tabular}{|c|c|c|c|}
\hline $\begin{array}{l}\nu \\
(\mathrm{GHz})\end{array}$ & $\begin{array}{c}\lambda \\
(\mathrm{cm})\end{array}$ & $\begin{array}{c}T_{\mathrm{CMB}}^{\mathrm{th}} \\
(\mathrm{K})\end{array}$ & Reference \\
\hline 19.0 & 1.58 & $2.78_{-0.17}^{+0.12}$ & Stokes et al., 1967 \\
\hline 20 & 1.5 & $2.0 \pm 0.4$ & Welch et al., 1967 \\
\hline 24.8 & 1.2 & $2.783 \pm 0.089$ & Johnson and Wilkinson, 1987 \\
\hline 30 & 1.0 & $2.694 \pm 0.032$ & Fixsen et al., 2004 \\
\hline 31.5 & 0.95 & $2.83 \pm 0.07$ & Kogut et al., 1996 \\
\hline 32.5 & 0.924 & $3.16 \pm 0.26$ & Ewing et al., 1967 \\
\hline 33.0 & 0.909 & $2.81 \pm 0.12$ & De Amici et al., 1985 \\
\hline 35.0 & 0.856 & $2.56_{-0.22}^{+0.17}$ & Wilkinson, 1967 \\
\hline 37 & 0.82 & $2.9 \pm 0.7$ & Puzanov et al., 1968 \\
\hline 53 & 0.57 & $2.71 \pm 0.03$ & Kogut et al., 1996 \\
\hline 83.8 & 0.358 & $2.4 \pm 0.7$ & Kislyakov et al., 1971 \\
\hline 90 & 0.33 & $2.46_{-0.44}^{+0.40}$ & Boynton et al., 1968 \\
\hline 90 & 0.33 & $2.61 \pm 0.25$ & Millea et al., 1971 \\
\hline 90 & 0.33 & $2.48 \pm 0.54$ & Boyton and Stokes, 1974 \\
\hline 90 & 0.33 & $2.60 \pm 0.09$ & Bersanelli et al., 1989 \\
\hline 90 & 0.33 & $2.712 \pm 0.020$ & Schuster et al., 1993 \\
\hline 90.3 & 0.332 & $<2.97$ & Bernstein et al., 1990 \\
\hline 90 & 0.33 & $2.72 \pm 0.04$ & Kogut et al., 1996 \\
\hline 154.8 & 0.194 & $<3.02$ & Bernstein et al., 1990 \\
\hline 195.0 & 0.154 & $<2.91$ & Bernstein et al., 1990 \\
\hline 266.4 & 0.113 & $<2.88$ & Bernstein et al., 1990 \\
\hline
\end{tabular}

G. Morigi, Bologna, G. Bonelli, Milano, J. B. Costales, UCB, Michel Limon, UCB, Yoel Rephaeli, Tel Aviv.

\section{The cosmic background explorer (COBE) mission}

In 1976 NASA formed the COBE Science Study Group consisting of Sam Gulkis from JPL, Michael

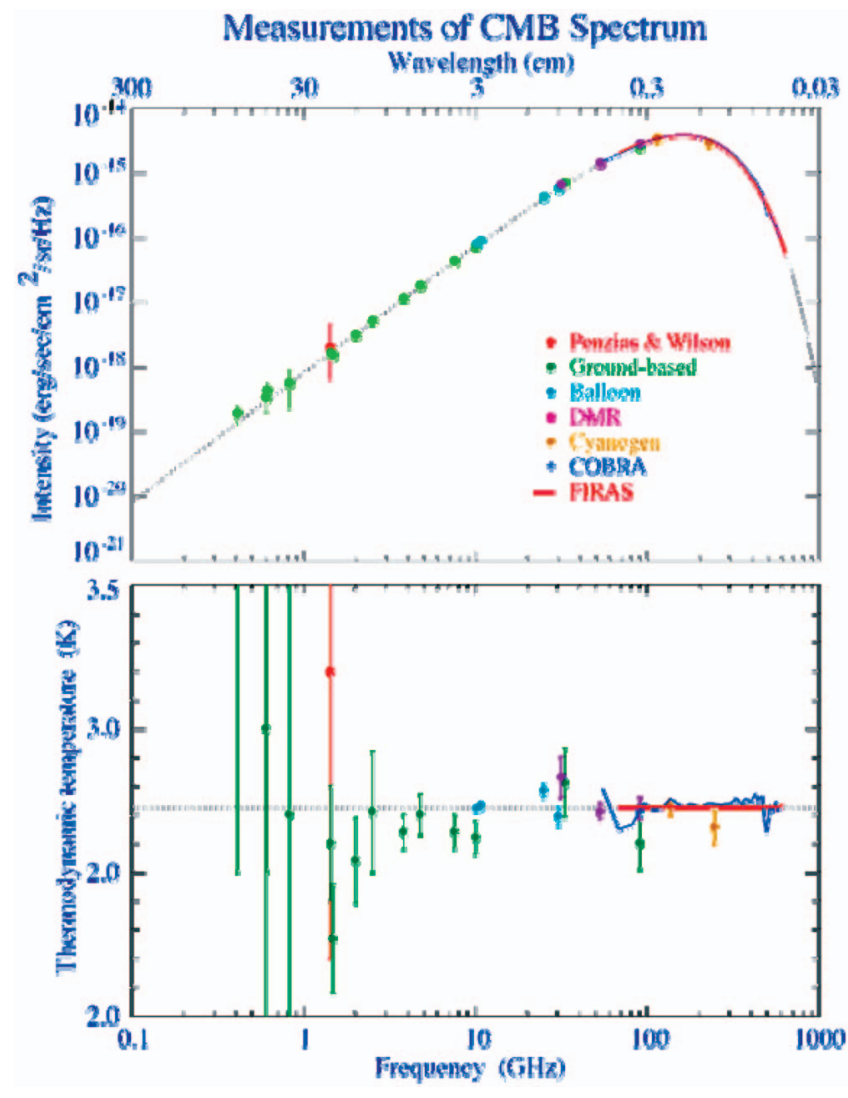

FIG. 5. (Color) Selected observations of the CMB frequency spectrum.

Hauser (P.I. for DIRBE Instrument) and John Mather (P.I. for FIRAS Instrument) from GSFC, George Smoot (P.I. for DMR Instrument) from SSL/LBL/UC Berkeley, Rainer Weiss, the chair, from MIT, and Dave Wilkinson from Princeton.

After many years additional scientists were added to form the COBE Science working group in the 1980s. These included Chuck Bennett, GSFC, Nancy Boggess,

TABLE IV. CMB temperatures as measured through the molecules CN.

\begin{tabular}{|c|c|c|c|c|}
\hline $\begin{array}{l}\nu \\
(\mathrm{GHz})\end{array}$ & $\begin{array}{c}\lambda \\
(\mathrm{cm})\end{array}$ & $\begin{array}{c}T_{\mathrm{CMB}}^{\mathrm{th}} \\
(\mathrm{K})\end{array}$ & $\begin{array}{l}\text { Observed } \\
\text { star }\end{array}$ & Reference \\
\hline 113.6 & 0.264 & $2.70 \pm 0.04$ & z Per & Meyer and Jura, 1985 \\
\hline 113.6 & 0.264 & $2.74 \pm 0.05$ & z Oph & Crane et al., 1986 \\
\hline 113.6 & 0.264 & $2.76 \pm 0.07$ & HD21483 & Meyer et al., 1989 \\
\hline 113.6 & 0.264 & $2.796_{-0.039}^{+0.014}$ & $\zeta \mathrm{Oph}$ & Crane et al., 1989 \\
\hline 113.6 & 0.264 & $2.75 \pm 0.04$ & $\zeta$ Per & Kaiser and Wright, 1990 \\
\hline 113.6 & 0.264 & $2.834 \pm 0.085$ & HD154368 & Palazzi et al., 1990 \\
\hline 113.6 & 0.264 & $2.807 \pm 0.025$ & 16 stars & Palazzi, 1992 \\
\hline 113.6 & 0.264 & $2.729_{-0.031}^{+0.023}$ & 5 stars & Roth et al., 1993 \\
\hline 227.3 & 0.132 & $2.656 \pm 0.057$ & 5 stars & Roth et al., 1993 \\
\hline 227.3 & 0.132 & $2.76 \pm 0.20$ & $\zeta$ Per & Meyer and Jura, 1985 \\
\hline 227.3 & 0.132 & $2.75_{-0.29}^{+0.24}$ & $\zeta \mathrm{Oph}$ & Crane et al., 1986 \\
\hline 227.3 & 0.132 & $2.83 \pm 0.09$ & HD21483 & Meyer et al., 1989 \\
\hline 227.3 & 0.132 & $2.832 \pm 0.072$ & HD154368 & Palazzi et al., 1990 \\
\hline
\end{tabular}




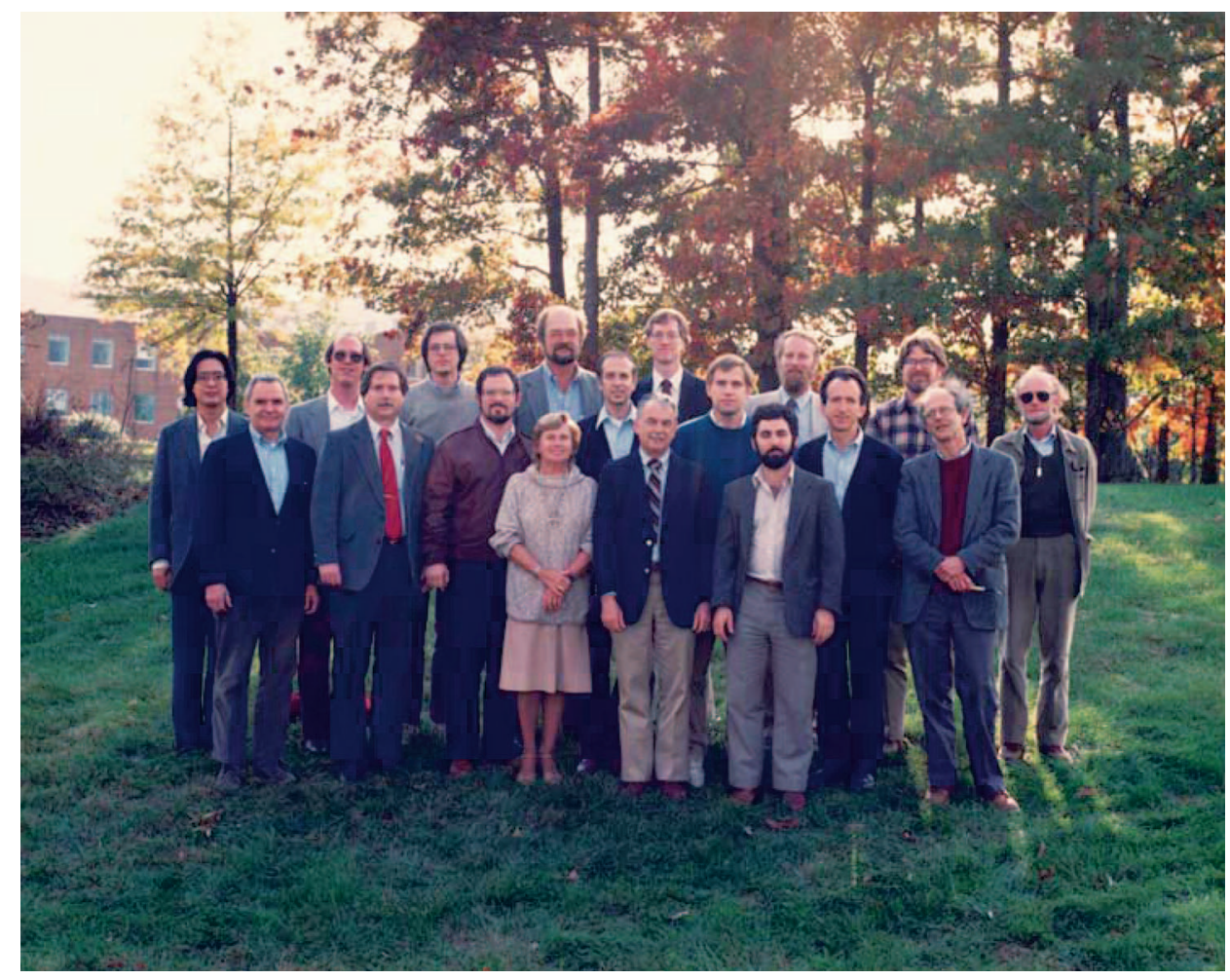

FIG. 6. (Color) Photograph of the COBE Science Working Group (minus Eli Dwek) taken during a meeting in 1988. From left to right, back row: Ed Cheng, Rick Schafer, Stephan Meyer, Mike Janssen, John Mather, Ned Wright, George Smoot, Tom Kelsall; middle row: Dave Wilkinson, Tom Murdock, Chuck Bennett, Bob Silverberg, Harvey Moseley, Michael Hauser, Rainer Weiss; front row: Nancy Boggess, Sam Gulkis, Phil Lubin.

NASA/GSFC, Ed Cheng, GSFC, Eli Dwek, GSFC, Mike Janssen, JPL, Phil Lubin, UCSB, Stephan Meyer, U. Chicago, Harvey Moseley, GSFC, Tom Murdock, General Research Corp, Rick Shafer, GSFC, Bob Silverberg, GSFC, Tom Kelsall, GSFC, and Ned Wright, UCLA (see Fig. 6).

At the same time that the science portion of the team was developing, the management, engineering, technical, and other mission support personnel were also developed. On November 18, 1989, after long preparation and delays, NASA launched its first satellite, the COBE, dedicated to cosmological observations. COBE had three scientific instruments:

(i) The far-infrared absolute spectrophotometer (FIRAS) to measure the CMB spectrum over the wavelength range $100 \mu \mathrm{m}<\lambda<1 \mathrm{~cm}$ with a $7^{\circ}$ resolution in order to investigate the blackbody nature of the $\mathrm{CMB}$ spectrum. It is designed to compare the spectrum of the $\mathrm{CMB}$ with that of a precise blackbody to measure tiny deviations from a blackbody spectrum.

(ii) The diffuse infrared background experiment (DIRBE) to map the spectrum over the wavelength range $1.25<\lambda<240 \mu \mathrm{m}$ in ten broad frequency bands with a $0.7^{\circ}$ resolution to carry out a search for the cosmic infrared background (CIB). CIB measurements are designed to measure visible light from very distant, unresolved galaxies. Light from all such distant galaxies is redshifted due to the cosmological expansion of the universe. The visible light from the galaxies is redshifted into the near infrared bands or absorbed by dust and reradiated in the far infrared and redshifted into the submillimeter band. The CIB measurements constrain models of the cosmological history of star formation and the buildup over time of dust and elements heavier than hydrogen.

(iii) The differential microwave radiometers (DMR) to map the $\mathrm{CMB}$ anisotropy in three frequency bands, $31.5,53$, and $90 \mathrm{GHz}$, with a $7^{\circ}$ resolution and a sensitivity better than one part in 100000 of the cosmic background temperature (see Fig. 3). The primitive form of the DMR was utilized in the 1940 s by Robert Dicke at Princeton University (see Fig. 7). The DMR does not measure the absolute temperature of a given direction of sky. Instead it measures the difference of temperatures of two different directions. A symmetrical DMR is one where two antennas pick signals from different directions and measure the difference between them. The two antennas quickly interchange positions and repeat the measurement. If the signals are of instrumental origin, they do not depend on direction, and the difference would not change its sign, but if the signals are of cosmological origin, the difference should change its sign when the antennas views are swapped. This operational scheme greatly reduces the systematic problems and improves reliability. The anisotropy map, a map of temperature difference, provides a snapshot of the universe at the time of recombination about 380000 years after the big bang. The map shows the primordial structures which could not have been affected by any physical process no faster than the speed of light by the recombination era. Many cosmological parameters that describe the dynamics and geometry of universe and initial conditions of big bang cosmology can be estimated from the anisotropy map. 

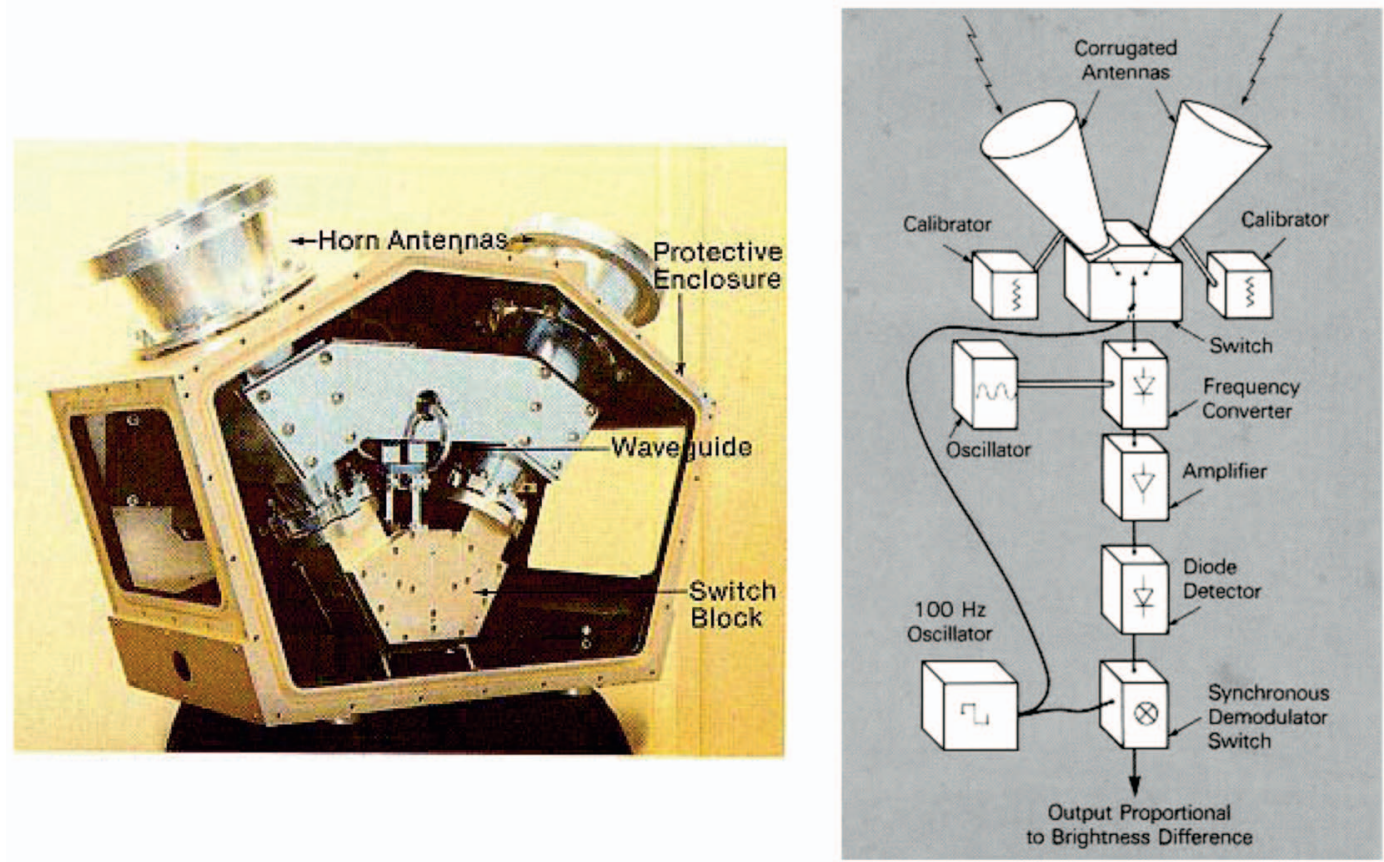

FIG. 7. (Color) The DMR instrument design. Left, photograph of the DMR instrument; right, schematic of the DMR. The DMR has played a key role in the CMB anisotropy experiments, including the U2 flight experiments and the COBE DMR. The WMAP also adopted differential radiometers as the basic apparatus for the CMB observations (http://lambda.gsfc.nasa.gov/product/cobe/).

\section{COBE results}

The COBE project was remarkably successful. FIRAS measurements corroborated the blackbody nature of the CMB spectrum (see Fig. 8), giving the background temperature (Mather et al., 1994), $T_{0}=2.726 \pm 0.010 \mathrm{~K}$, $95 \%$ C.L. The DIRBE instrument provided absolutely calibrated maps of the sky at many wavelengths, gave an unparalleled picture of our own galaxy, and a good measurement of the cosmic infrared background radiation which is the radiation from the first generation of stars (see Fig. 9). Most of this radiation comes from early, bright, dusty galaxies.

The FIRAS observations established very strongly that the $\mathrm{CMB}$ is the relic thermal radiation from the big bang and the DIRBE results revealed more about the later universe. The DMR discovery of CMB anisotropies got the most attention and has led to a whole area of activity with much theory, additional experiments,

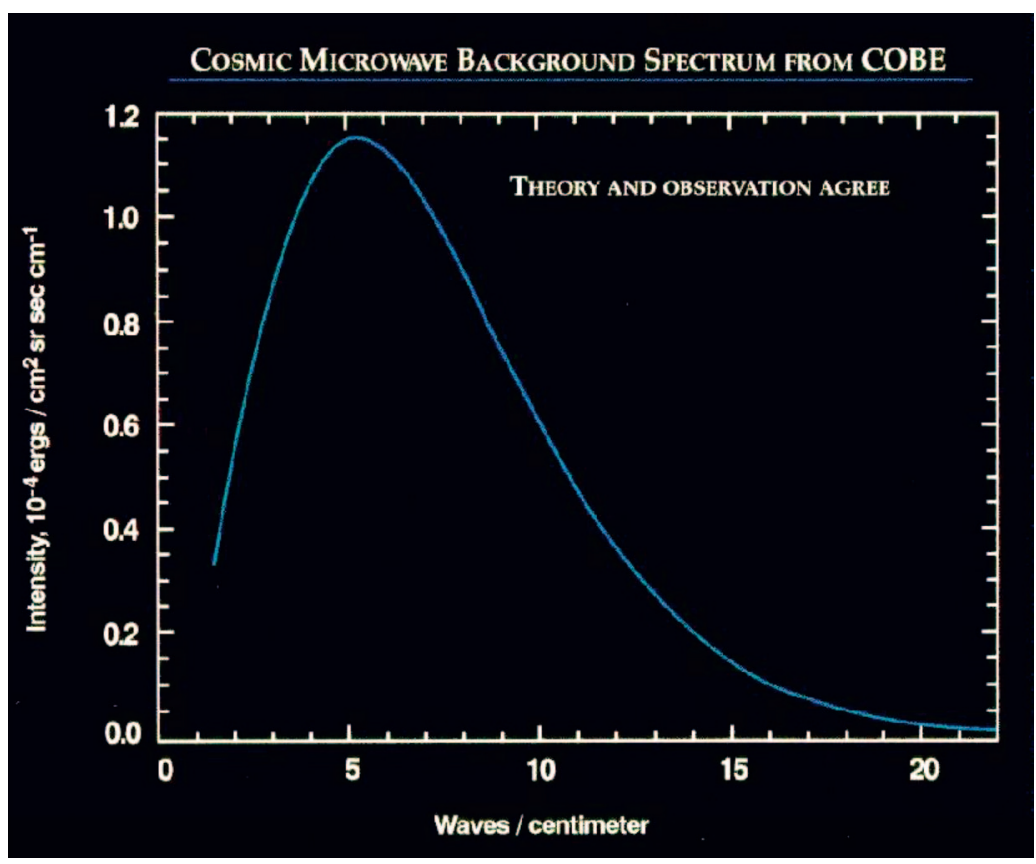

FIG. 8. (Color) The solid (blue) curve shows the expected intensity from a single temperature blackbody spectrum, as predicted by the hot big bang theory. The FIRAS data fit with the expected blackbody curve with $T$ $=2.726 \mathrm{~K}$ so precisely that the uncertainties are smaller than the width of the blackbody curve, the data points are covered by the curve and not visible (Mather et al., 1994). 


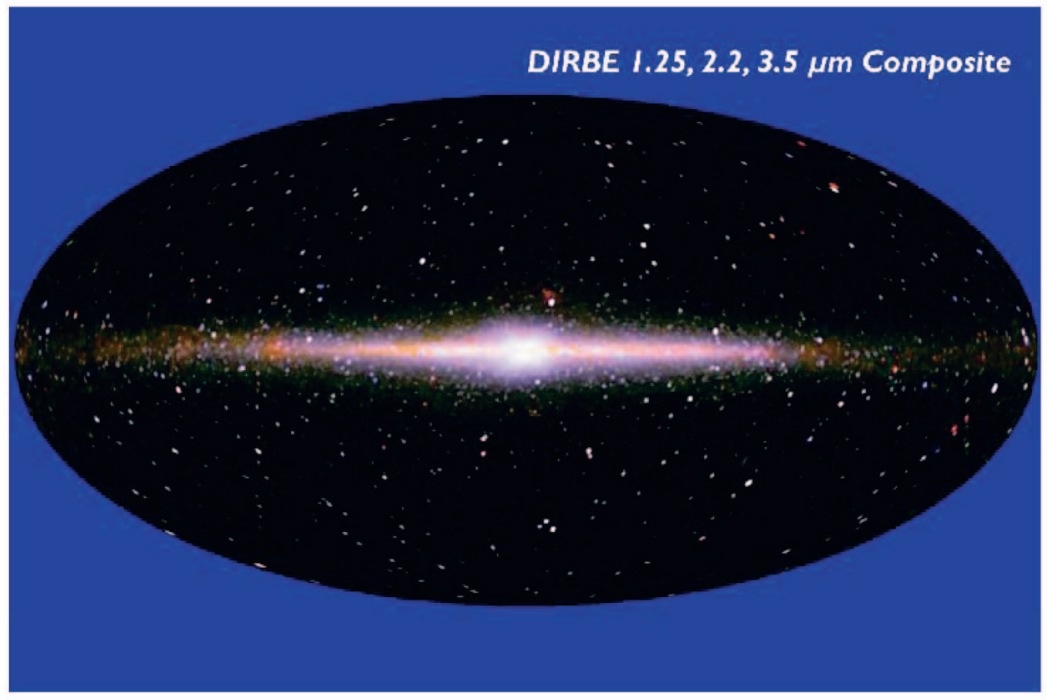

FIG. 9. (Color) False-color image of the nearinfrared sky map $(1.25,2.2$, and $3.5 \mu \mathrm{m}$ composited) mapped by DIRBE. The dominant sources of infrared light at these wavelengths are stars in the Milky Way, as shown by the thin disk and the bulge at the center. Scattered bright sources can also be seen off the galactic disk (http://lambda.gsfc.nasa.gov/ product/cobe/).

and space missions. The discovery of CMB anisotropies was a many step process that involved the development of the instrumentation and techniques including calibration as well as the development of software and personnel to construct the instrument, carry out the observations, process, and then finally analyze the data. To this point we have seen the historical development of some of these. For the COBE DMR the development of the experiment including instrumentation, calibration, software, and personnel was highly integrated though often in time order due to the long period which COBE took. The outstanding instrumentation portion of the team, e.g., Roger Ratliff (microwave components), John Maruschak (component testing and verification), Robert Patschke (31.4 GHz), Maria Leche $(53 \mathrm{GHz})$, Larry Hillard $(90 \mathrm{GHz})$, Cathy Richards (receiver upgrade), Rick Mills (test chamber and organization), Peter Young (test results), Marco Toral (antennas), Gene Gochar (mechanical engineering), Frank Kirschman (thermal design), Dave Amason (command procedures), Chris Witebsky (90 GHz design), Dave Nace and Bernie Klein (instrument engineer), Dick Weber (systems engineer), and so on, tested and assembled components, subsystems and then the full set of DMRs were tested and calibrated. Goddard had been fairly restricted in hiring and chose to do COBE as an in-house project and thus was able to make additional hires so many young people were hired and trained on this project. It was very rewarding working with these people and to see their development and eagerness to take on challenging work and significant responsibilities.

During the end of this period, the data processing and analysis team began building a data processing pipeline with simulations and systematic checks and reviews. Sergio Torres, Jon Aymon, Charles Backus, Laurie Rokke, Phil Keegstra, Chuck Bennett, Luis Tenorio, Ed Kaita, R. Hollenhorst, Dave Hon, Qui Hui Huang, Al Kogut, Gary Hinshaw, Robert Kummerer, Jairo Santana, Kris Gorski, Tony Banday, Charley Lineweaver, Giovanni De Amici, Pete Jackson, Kevin Galuk, Vijay Kumar, and Karen Loewenstein were the many people who worked on the DMR data processing and analysis. This is, of course, a very small fraction of the people who worked on COBE over the many years.

We started with a small team of five post docs and one graduate student developing the software about one and a half years before launch. We built up the team slowly as launch approached and passed. About half an hour after COBE was launched it flew over Antarctica and the extra ice-reflected sunlight produced excess solar electric power and mission control asked to turn on the DMRs earlier than their original timeline to absorb some of the excess power. I quickly approved and the DMR instruments were commanded on. As soon as the DMRs power came on, the internal noise calibrators fired as they were programmed, producing a nice strong but on scale signal, then as the COBE space craft rotated, the DMR beams swept over the moon (lucky alignment) and we could tell from our real-time software plots that the DMRs had survived the launch and were operating apparently nominally given the observed signals and apparent noise. Only time would tell if the instrument would remain sufficiently stable and reliable to make the required observations.

At the end of the first day, Sergio Torres and Dave Hon ran the full map-making pipeline and it worked well. We had DMR maps covering half the sky. They were noisy but still the best maps anyone had to that point. We were all in good spirits and filled with optimism for the long period of observations and processing that was going to be necessary. After six months we had a substantial amount of data and things were working well enough that we made a full, carefully documented map pipeline run and produced maps covering essentially the full sky. These maps were quite good and had what we know in retrospect was just enough sensitivity to detect the primordial CMB anisotropies. We did note that there were small significant effects in the data but chose to publish only an upper limit on anisotropies to be conservative since we had only limited time to understand fully the experiment and there were possible systematics in the instrument, our estimate of the possible 
astrophysical foregrounds, or in the data processing and analysis. We knew that we could make significant improvements in the processing of the data and correct for small residual effects. We wanted also to do sensitivity studies to ensure that we understood the experiment well.

During my training as a young scientist, my mentors, including Dave Frisch, Luis Alvarez, and their colleagues, repeatedly emphasized the importance of maintaining high scientific standards, both in choosing good projects and in executing them well. I heard of how important it was in this kind of experiment to set up systems and approaches so that one does not mislead oneself and assume that one has things right. Often even the most careful person lets down their vigilance when the results are coming out as expected and pays more attention when the result is different or unexpected. This is simply human nature and one must be careful not to fall in this trap. There was a famous paper showing the measurements of the speed of light with error estimates over time. It systematically changes with time but almost always within the estimated errors even though over many experiments, it changes by many standard deviations. This was unequivocal proof of this tendency and thus the need to be rigorous and systematic regardless of the result one was obtaining. One should work until confident in how well one has done before looking at the result and start believing in it. In many fields it is essential procedure to do the experiment in a double-blind method so as to guard against this very expectation effect.

So as soon as we had revamped and retested our data processing and map-making pipeline, we carefully validated it and then processed the full first-year data set. When the maps were made, there were clearly some residual signals above the noise. A sure-fire indicator was that when we made maps at each DMR frequency (31.4, 53 , and $90 \mathrm{GHz}$ ) to get the best sensitivity we co-added the data from each of the two (A and B) channels at each frequency. However, we could also difference the A and B maps which should subtract any sky signal and leave only the independent instrumental noise. The variances and the smoothed $\mathrm{A}+\mathrm{B}$ maps looked significantly different from the A-B maps. The A-B maps were consistent with what was expected from the simple Gaussian fluctuations of the receiver white noise. The question was: Were these extra signals from the CMB, from astrophysical sources, from the instrument and experimental procedures, or from the data processing? At one of our COBE science team meetings I reported to the full Science Working Group (SWG) that we saw some effects but that it was too soon to be sure that it was not some artifact. I set the goal for the DMR analysis team of systematically going through all our steps and processes to show that we had each thing accounted for properly since in this kind of experiment one attributes to the $\mathrm{CMB}$ everything that is not attributed to anything else. We set up a plan and schedule to work through everything systematically and have each study confirmed and checked. The team fell into hard focused work on this program not jumping right to the conclusion that this was CMB anisotropy but assuming that this was the correct approach. About six months (August 1991) into this, Ned Wright turned part of his attention to the DMR and noted that the one-year DMR maps had fluctuations in them consistent with the cold dark matter (CDM) cosmological model. This did not deflect the DMR team much as they were still trying to get done the projects set for them by their deadlines. In October, Ned Wright did another bit of processing of the one-year maps with software he wrote and concluded that the data fit a Harrison-Zel'dovich spectrum with a corresponding quadrupole amplitude of about $15 \mu \mathrm{K}$. We found about $30 \mu \mathrm{K}$ in the DMR official software. Ned was now excited about the DMR potential results and wanted to bring it up at the next science team meeting. We agreed to have it at a more private special evening meeting after dinner at $\mathrm{Al}$ and Nancy Boggess's house as it would be a sensitive issue. The WG (as I argued) thought it appropriate to be conservative, educate all the members of the team on the status and reliability of the results, and have the DMR team prepare and present to the full science team a review of all portions of the DMR experiment. This review was set for the end of the year 1991.

This extra attention from the SWG began to affect the DMR analysis team. They did not want to miss out on the discovery and especially the fun and excitement of the science analysis and the cosmological implications. I tried to have no one work on science analysis papers (myself included) until the checks were done. This was for the very point of ensuring that we had done a job that we were very confident was as good as possible. I worked hard to keep them on track and careful about getting the correct results and went so far as to offer two free tickets anywhere in the world to any one that could find an effect from anything (other than the CMB) what would explain a significant portion of what we were seeing. We divided into specialists in the areas of instrumental errors (led by the outstandingly able Alan Kogut who had to deal with very many potential issues), galactic foregrounds (Chuck Bennett and Gary Hinshaw), software (Sergio Torres for the DMR official code, George Smoot and my graduate students Luis Tenorio and Charley Lineweaver creating independent code and tests, and Ned Wright with his own map processing software), and so forth. David Wilkinson was a particularly determined skeptic keeping everyone on their toes.

We held the review just after New Year's and presented all the tests, checks, calibrations, and results in a day long review with extra material to the full DMR and SWG. There were a few things left to follow up but with them underway, we turned to analyzing the maps for their scientific results. There are a number of interesting things here including the low quadrupole and so forth but the most important issue was following a rigorous procedure. This was evident to me when we had the last SWG meeting to review the results and papers and discuss going public. At that meeting we also heard from the MIT-Princeton team with a balloon-borne bolometer 
experiment whose data seemed to support the DMR results though were not as strong as the DMR. That seemed consistent but not conclusive. Then Phil Lubin got up and argued against going public stating that his group's data from their South Pole experiment was inconsistent and had a lower upper limit than the DMR results. This caused concern among some of the SWG but I found myself confidently arguing for going forward and publishing. I felt that the DMR team had done about as good a job as one could and were very likely to be correct.

On April 23, 1992, the COBE team announced the historical discovery of the anisotropies of cosmic microwave background radiation with characteristic anisotropy $\Delta T / T \approx 10^{-5}$ or $\Delta T \sim 30 \mu \mathrm{K}$ on angular scales larger than $\sim 7^{\circ}$ at the annual meeting of American Physical Society in Washington, D.C. (Smoot et al., 1992). The CMB anisotropy provides very rich information on the early universe, allowing the calculation of cosmological parameters and discrimination of various detailed models of the big bang. The anisotropy map produced by the COBE DMR is composed of 6144 pixels each $2.6^{\circ} \times 2.6^{\circ}$. This can be compared to the size of a patch of sky subtended by light that had been traveling since the beginning of the universe, about $1^{\circ}$. That is, the perturbations detected by DMR are directly from the primordial state set at the beginning. After four years of measurements by the COBE DMR, the typical signalto-noise ratio in a $10^{\circ}$ smoothed frequency-averaged map rose to $\sim 2$, so the anisotropy could be seen by eye. The DMR found the CMB thermodynamic temperature of $T_{0}=2.725 \pm 0.020 \mathrm{~K}$ which was well consistent with the result of COBE FIRAS. If we subtract this temperature from the map and change the scale by a factor of 1000 , the $\mathrm{CMB}$ dipole looms out of the uniform background with amplitude $3.358 \pm 0.024 \mathrm{mK}$ toward the galactic coordinates $\left(l^{G}, b^{G}\right)=\left(264.31 \pm 0.16^{\circ}, 48.05^{\circ} \pm 0.10^{\circ}\right)$ reconfirming the discovery of U2 flight experiments. When the scale is increased to 100000 , higher multipoles ( $l$ $\geq 2$ ) can be seen. Figure 10 shows these features at these increasing scale factors. The quadrupole amplitude was estimated between 4 and $28 \mu \mathrm{K}$. The analysis of multipoles with $l>2$ showed that the fluctuations are consistent with scale invariant (an $n=1$ power-law) fluctuation spectrum as predicted by models of the inflationary big bang (Fig. 11). Another important test for the inflationary big bang theory is the Gaussian distribution of the primordial temperature fluctuations. Our COBE DMR data found no evidence for deviations from a Gaussian distribution (Smoot et al., 1994) as have all the experiments that have followed thus far.

\section{FORGING THE STANDARD MODEL OF COSMOLOGY: $\Lambda$ CDM}

\section{A. The suborbital CMB experiments}

The CMB anisotropy is the most important cosmological observable to date, so many more ground-based and balloon-borne $\mathrm{CMB}$ experiments followed the

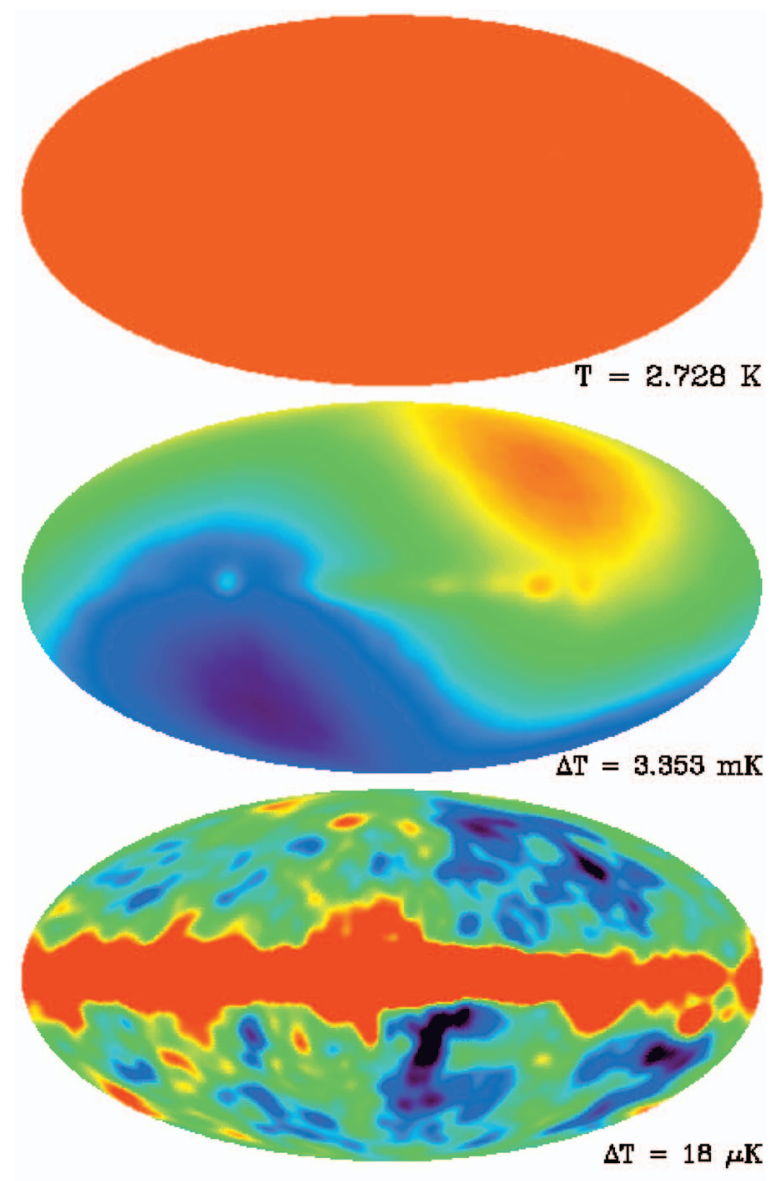

FIG. 10. (Color) The breakdown of the CMB sky map produced by COBE DMR (http://lambda.gsfc.nasa.gov/product/ cobe/), monopole (top), dipole (middle), and multipole (bottom). At $T \sim 3 \mathrm{~K}$ level (top), we have uniform radiation from every direction. When the sensitivity is changed to $\sim \mathrm{mK}$ level, the dipole pattern shows up, which is due to the peculiar motion of our solar system relative to the CMB rest frame. As the scale is refined to $\Delta T \sim 10 \mu \mathrm{K}$, after removal of the dipole, the multipole features of anisotropy become evident. These very tiny fluctuations give us information about the early universe.

COBE mission in 1990s (Smoot et al., 1997). While some experiments focused on large angular scales at frequency bands not used in the COBE DMR, most of the projects worked on the smaller angular scales which were not explored by the COBE DMR. These small angular scale experiments put very tight constraints on cosmological models. Results from some representative experiments on the angular power spectrum are presented in Fig. 12. Supernovae observations startled the cosmology community with the discovery of accelerated expansion of space (Riess et al., 1998; Perlmutter et al., 1999). This brought back the cosmological constant to the Einstein equations, introduced originally with different motivation and then discarded soon after. Observations other than the CMB experiments including galaxy surveys have provided valuable information and allowed cross checks with the results from the CMB experiments. Some recent results of suborbital experiments 


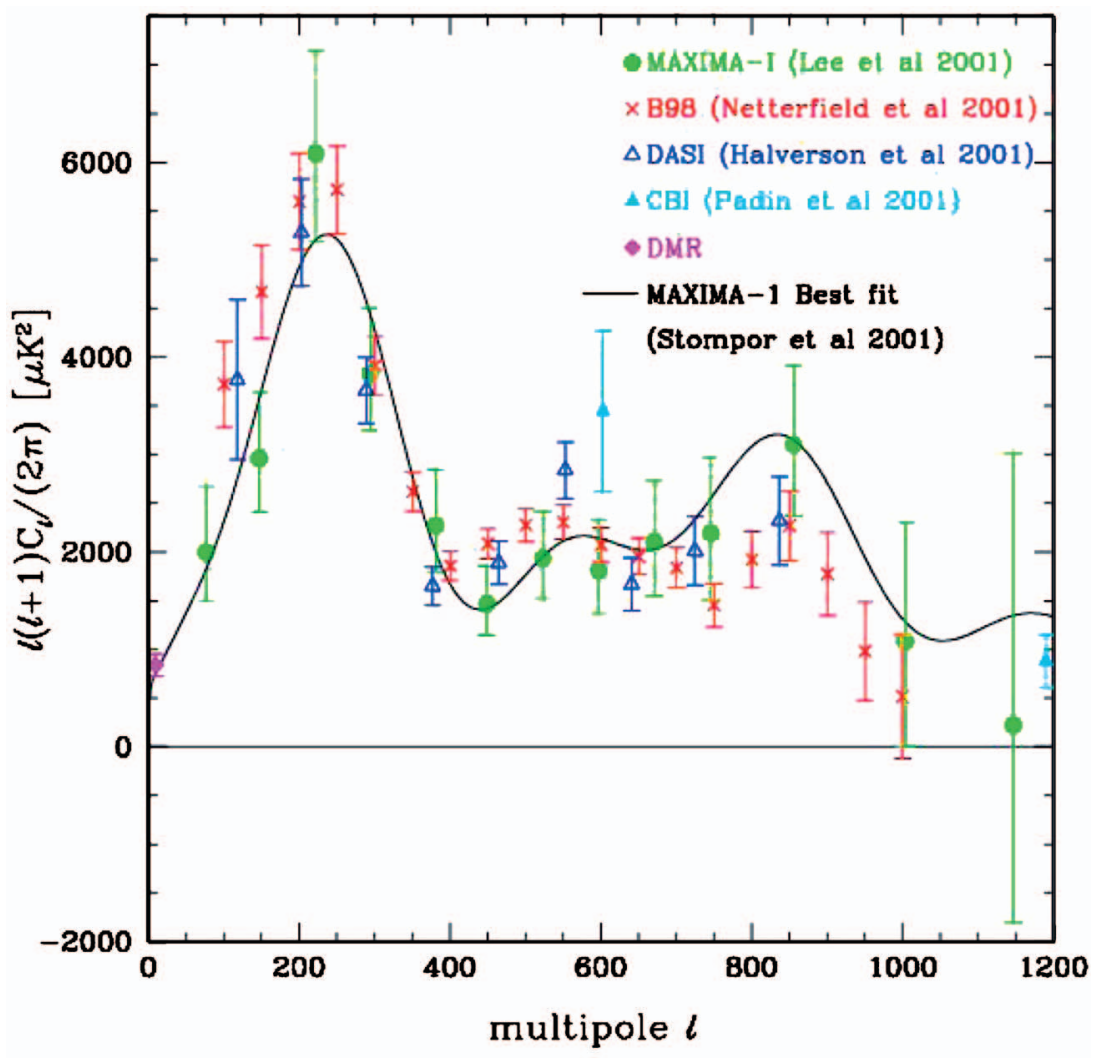

FIG. 11. (Color) By 2001 the first acoustic peak was observed and there was evidence mounting for a second peak in the $\mathrm{CMB}$ angular power spectrum based upon the observations obtained from COBE DMR, MAXIMA, BOOMERanG, DASI, and CBI. In a couple more years much more data were available and the WMAP first year data then traced out the plateau and first peaks. The solid blue line is the prediction curve of a model with $n=1, H$ $=50 \mathrm{~km} / \mathrm{s} \mathrm{Mpc}$ and cold dark matter (http://cosmas.lbl.gov/).

and observations are presented in Table V, Fig. 12, and Fig. 17.

\section{B. Physics from CMB anisotropy power spectrum}

The observations of the CMB anisotropy power spectrum have turned us from speculations about the universe with meager measurements to a working cosmological model in which the universe is spatially flat, consists mainly of dark matter, and a small fraction of ordinary matter just sufficient to produce the light element primordial abundances. All the complex (cosmic web) structure of matter formed from primordial adiabatic fluctuations believed to be the result of quantum mechanical fluctuations from when the universe was a tiny fraction of a second old-the inflationary epoch. Observations to date have achieved of order a few percent accuracy on the key cosmological parameters and

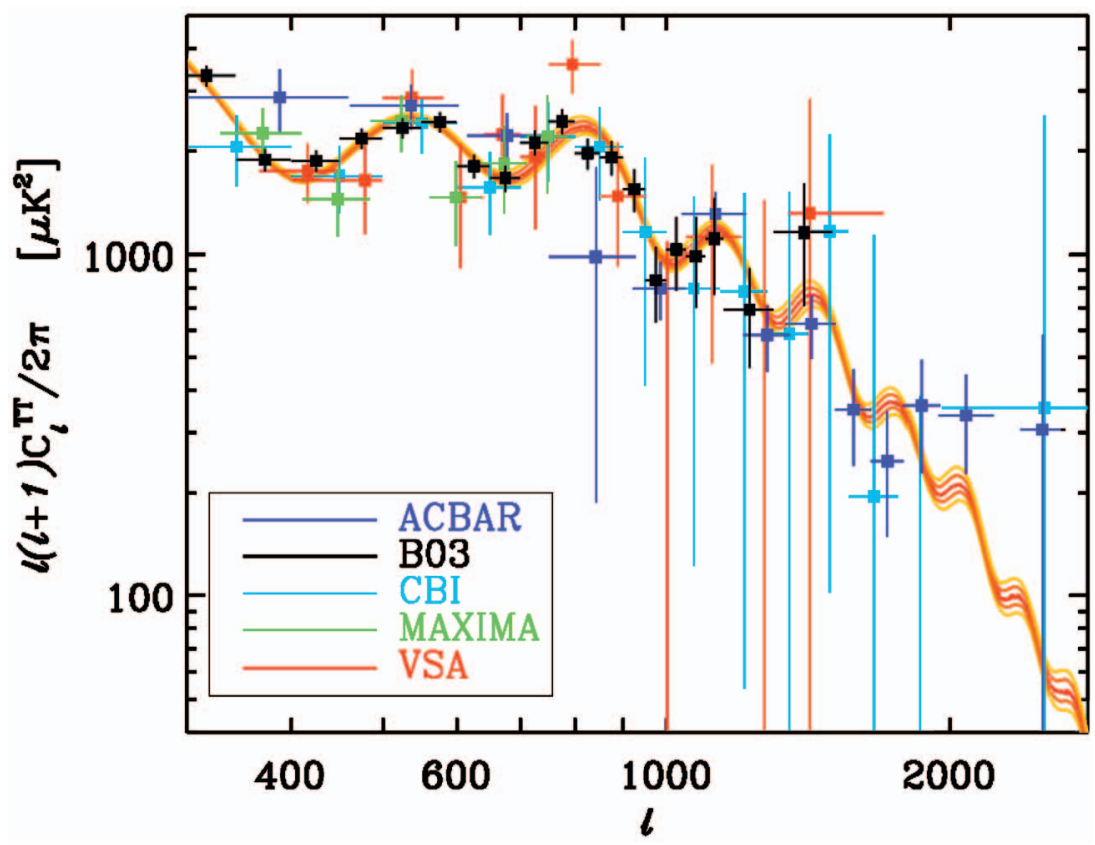

FIG. 12. (Color) The best fit curve (orange) of small-scale angular power spectrum for $\Lambda \mathrm{CDM}$ model estimated with WMAP data and measurements carried out by groundbased and balloon-borne CMB experiments (Spergel et al., 2006). Notice that this shows the greater details of angular power spectrum at the range $l$ $\geq 300$. 
TABLE V. Cosmological parameters for $\Lambda$ CDM model. $\Lambda$ CDM parameters computed using WMAP 3 -year data only (middle column) and various data sets combined (right column). $\Omega$ refers to the density relative to the critical density.

\begin{tabular}{lcc}
\hline \hline Parameter & WMAP 3-year & All combined $^{\mathrm{a}}$ \\
\hline$H_{0}$ (Hubble parameter) & $73.5 \pm 3.2 \mathrm{~km} / \mathrm{s} / \mathrm{Mpc}$ & $70.8_{-1.6}^{+1.5} \mathrm{~km} / \mathrm{s} / \mathrm{Mpc}$ \\
$h$ (reduced Hubble parameter) $^{\text {(scalar spectral index) }}{ }^{\mathrm{b}}$ & $0.735 \pm 0.032$ & $0.708_{-0.016}^{+0.015}$ \\
$n_{b} h^{2}$ (baryon density) & $0.951 \pm 0.016$ & $0.938_{-0.014}^{+0.015}$ \\
$\Omega_{\Lambda}$ (dark energy density) & $0.0223 \pm_{-0.00073}^{+0.00075}$ & $0.02193_{-0.00068}^{+0.0006}$ \\
$\Omega_{m}$ (matter density) & $0.763 \pm 0.034$ & $0.738 \pm 0.016$ \\
$\sigma_{8}$ (matt. fluc. On $\left.8 h^{-1} \mathrm{Mpc}\right)$ & $0.237 \pm 0.034$ & $0.262 \pm 0.016$ \\
$t_{0}$ (age of the universe) & $0.742 \pm 0.051$ & $0.751_{-0.031}^{+0.032}$ \\
$\tau$ (reionization optical depth) & $13.73_{-0.15}^{+0.16} \mathrm{Gyr}$ & $13.84 \pm 0.14 \mathrm{Gyr}$ \\
\hline \hline
\end{tabular}

${ }^{a}$ Results with combined data from the experiments WMAP, 2df, SDSS, BOOMERanG, ACBAR, CB I, VSA, SN astier, SN gold, WL, and BAO.

${ }^{\mathrm{b}}$ At $k=0.002 / \mathrm{Mpc}$.

the coming decade is likely to see this accuracy improve to less than a percent. In large part, this is due to the expected improvement in $\mathrm{CMB}$ temperature and polarization anisotropies. That change will make a very substantial difference in our understanding and in our ability not only to determine those parameters but also to test the key elements of the assumed physics of our cosmological model and even probe what were the natural and minimum initial conditions.

These new results, their analysis and interpretation, will have profound implications for physics and astronomy. The current successful models call for four major new pieces of physics: dark energy, dark matter, inflation (or alternative), and the matter-antimatter asymmetry and assume four other major items: no other significant relics of the early universe, there are no significant extra dimensions, the fundamental constants do not vary with time, and there are no other significant exotic forces in play. All of these will be tested and probed in a precise way.

An important reason for this is the physical simplicity of the early universe and processes that leave their imprint on the CMB. The CMB is a very rich source of information because all oscillations are still linear and the physics of the fluid is well understood and there are many possible features to probe and observe.

The early universe is dominated by the cosmic background radiation (CBR) photons which interact strongly with the electrons (electrically coupled to protons and helium nuclei) to make what is called the photon-baryon plasma. This plasma undergoes simple acoustic oscillations until the universe cools enough for neutral atoms to form and the baryons and photons lose their tight coupling. The photons are then free to propagate across the universe from this last epoch plasma which marks the cosmic photosphere very much like the apparent surface of the sun (solar photosphere). The photons that were set free in the transition from a cosmic plasma to stable atoms make up the cosmic microwave back- ground radiation which is present everywhere in the cosmos. As we observe this radiation in the sky, we are practically looking at a snapshot of the early universe.

It is easy to calculate that this deionization happened when the universe was 1089 times smaller than the present at a time about 379000 years from the beginning of the big bang. The process is set by the adiabatic expansion of the universe which gives the ratio of CBR temperatures being the inverse ratio of universe scale sizes. Thus when the universe was 1089 times smaller than now, the CBR temperature was 1089 times higher or about $3000 \mathrm{~K}$ which because of the large ratio of photons to baryons is sufficient to ensure that the universe is fully ionized. (Go to the equilibrium equations of Saha and put in a hydrogen ionization energy of $13.6 \mathrm{eV}$ and the temperature of $3000 \mathrm{~K}$ or equivalent mean energy about $1 / 4 \mathrm{eV}$ per photon provides enough photons above $13.6 \mathrm{eV}$ to ionize all the hydrogen and helium.) This happens fairly rapidly but not instantaneously and thus extends over about $\Delta z \approx 70$ compared to 1089 which is similar to the skin on an apple. The finite thickness of the region of last scattering of photons with the primordial plasma is an important reason that there is a damping of the visibility of small features or the damping of the high spatial frequencies (high $l$ ). Now we can understand the roll off (diminishing of signal) at small angular scales.

\section{The geometry of space-time}

We can probe the geometry of space-time by observing sound waves in the primordial plasma. The CBR dominates the early universe and thus photons (and other relativistic particles) moving at the speed of light in random directions are the particles that make up most of any sound wave in the early universe. Because we live in three dimensions, the speed of sound will be the 


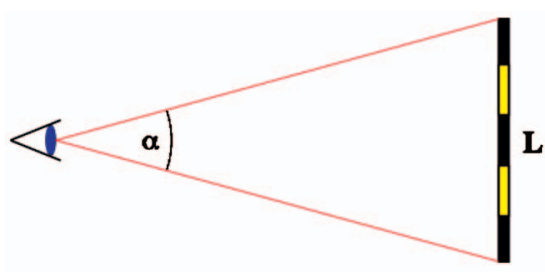

FIG. 13. (Color) Flat or Euclidean geometry.

speed of light divided by root $3\left(v_{s}=c / \sqrt{3}\right)^{2}$

According to Jeans' theory, first derived to explain the formation of the solar system and oscillations of stars under the influence of gravity, if the free fall time is shorter than the sound crossing time, gravitational collapse occurs. If the sound crossing time is shorter than the free fall time, then the system can adjust and undergoes acoustic oscillations. The sun oscillates as a result of any perturbation. So does the early universe, since the sound speed is so high and the gravitational perturbations are so small $\left(10^{-5}\right)$ that gravity is weak and the free fall time is very long. Primordial perturbations all start near time equals zero and oscillate acoustically-i.e., as sound waves. The CBR is freed at $1+z=1089$ or about 379000 years so that the CMB fluctuations image them at that epoch. Primordial perturbations have had essentially an elapsed time of 379000 years to oscillate at the speed of sound.

At the close of those 379000 years, the largest possible coherent acoustic oscillations had a spatial extent of roughly 220000 light years (or 70000 parsec). There was simply no time for more: With the speed of sound at 0.6 light speed and a time of 379000 years, the largest regions in which coherent oscillations could develop had a spatial extent of $0.6 \times 379000=220000$ light years. This upper limit is called the "sound horizon." One reason these oscillations are of great interest to cosmology is that $\mathrm{CMB}$ anisotropy observations can determine the apparent angular size of the sound horizon in the sky.

When the first stable atoms formed, the sound waves in the cosmic plasma caused tiny fluctuations in the temperature of the cosmic microwave background. The cosmic microwave background fluctuations were there before the atoms formed. Satellites WMAP and Planck and other suborbital instruments can map those temperature differences with high precision.

However, what is observed is not the absolute size of the sound horizon, but its angular size in the sky. We do know the absolute size already, namely the 220000 light years mentioned above. By comparing the angular and absolute sizes, we can determine the curvature of our cosmos-whether space is flat, or has a spherelike or

\footnotetext{
${ }^{2}$ At the last scattering surface the baryon density has increased to the point that it slightly affects the speed of sound. The baryon-photon momentum density ratio $R=\left(\rho_{b}+p_{b}\right) /\left(\rho_{\gamma}\right.$ $\left.+p_{\gamma}\right) \approx 0.6\left[\Omega_{b} h^{2} / 0.02091000 /(1+z)\right]$ so that $v_{s}=c / \sqrt{3(1+R)}$ $\simeq c / \sqrt{3\left(1+3 \rho_{B} / 4 \rho_{\gamma}\right)}$ and this causes a small correction to this estimate.
}

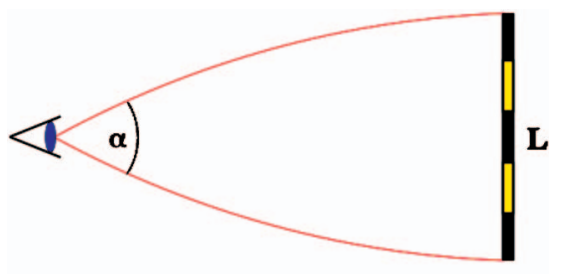

FIG. 14. (Color) Closed or positively curved geometry.

saddlelike shape. In ordinary Euclidean space ("flat space"), we are well acquainted with the relationship: The angular size (the "apparent size") of a given object decreases linearly with the distance-at least for faraway objects. Figure 13 shows the relationship between the spatial extent $L$ of a measuring rod, its distance, and its angular size $\alpha$. Shown in red in Fig. 13 are light rays connecting us, the observer, with both ends of the measuring rod.

Matters are somewhat different in a space of positive curvature, the three-dimensional analog of a spherical surface. In such a space, light does not travel along straight lines. Instead, light rays converge, as can be seen in Fig. 14. The angular size (the apparent size) of a given object decreases more slowly than linearly with the distance-at least for far-away objects. In such a space, the same measuring rod at the same distance from us will have a larger apparent size, corresponding to a larger angle $\alpha$.

In a space with negative curvature, it is the other way around: light rays diverge, and as a result the same measuring rod, still at the same distance, will have a smaller apparent size, corresponding to smaller $\alpha$ (see Fig. 15).

Consequently, it is simple to read off the curvature of space from the cosmic microwave background. We know the absolute size of the largest sound waves in the early universe, and we can measure their apparent size in the sky. The distance of the microwave background can be calculated. We know the temperature at which it was formed, and we can measure its temperature today. The temperature difference between then and now is directly related to the amount by which the universe has expanded from then until now, and this, in turn, is directly related to the distance. Comparing the apparent and true sizes at the calculated distance, we obtain the curvature of space. In this way, we have determined that, to high precision, space in our cosmos is flat.

\section{Acoustic oscillations}

So why do the acoustic peaks show up? What do they tell us? The geometry of space-time is most precisely

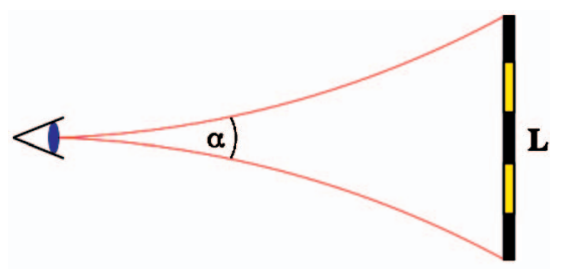

FIG. 15. (Color) Open or negatively curved geometry. 
determined by measuring the angular scale of the first acoustic oscillation peak in the angular power spectrum. With observations of several peaks we are able to tie down several parameters and determine the geometry of space-time even more precisely. How can we understand the plateau and the acoustic peaks?

That again is conceptually simple. If a physical system is hit by a spike of short duration-the extreme version is a Dirac delta function, i.e., an impulse of zero time duration - then all possible oscillation modes are excited equally on average but stochastically. This would be equivalent for the early universe of equal power or identical rms perturbation amplitudes into all plane wave for flat space-time or appropriate modes. So, if we choose the correct plotting of the angular power spectrum, the initial perturbations or excitation would be basically in dependent of scale and particularly angular frequency. The finite thickness of last scattering will damp out the high angular frequencies. Another effect which is leakage of photons out of high frequency perturbations because of minor edge effects adds to this effect. Because of the geometry of space-time and the contents of the universe, this otherwise nearly scale invariant power spectrum has a set of acoustic peaks at a fundamental angular frequency and its harmonics. These are really three-dimensional perturbations that are then manifested upon the apparently spherical sky.

On the largest angular scales, e.g., those first observed by the COBE DMR, we observe what are the basically initial conditions. These scales are so large that even moving at the full speed of sound $(c / \sqrt{3})$ the change is small compared to the size of the structure and elements that large have only undergone a very small fraction of an oscillation from the beginning until the last scattering surface. Perturbations that are much larger than the sound horizon remain little changed and will show up in the power spectrum as simply the low angular frequency (large angular scale) plateau that reflect the primordial perturbations. Without the acoustic oscillations, the angular power spectrum would remain flat until the high angular frequency (small physical scales) damping cuts it off.

Why is there a well-defined first peak, if all scales are simply oscillating and there is little damping on the larger scales?

However, when one reaches the scale of about 220000 light years at the surface and time of last scattering, the oscillation has had time to just fully compress. Compression makes that region hotter and that is what shows up in the snap shot provided by the CMB.

At half that size an acoustic oscillation has time to compress and then expand and reach maximum rarefaction. It would appear to be cooler than the average. In an angular power spectrum this would appear as the second peak, since taking the power rectifies (squares) the variation which was to cooler.

At one-third that size the acoustic oscillation has time to compress, rarify, and reach maximum compression again. This is the source of the third acoustic. And so on for higher spatial frequency peaks. It will appear warmer than the average. At 3/4 that size the oscillation has compressed and then expanded back to the starting place for no contrast to the average. Thus measuring the mean square fluctuations on each scale or the power spectrum, one would anticipate peaks at the sound horizon, half the sound horizon, one-third the sound horizon, etc., and then nulls midway in between. However, there is an additional effect from the motion of the oscillations-at the halfway points the sound wave is moving with maximum speed and the Doppler effect produces a small secondary peak exactly out of phase with the compression and rarefaction. These somewhat fill in between the acoustic peaks so that there is not a precise null.

The key points are that (i) compressed fluid is hotter, (ii) oscillation frequency scales inversely with size, (iii) oscillations stop at last scattering, (iv) nulls of oscillations are when the Doppler effect is maximal from the motion of oscillation, and (v) fluctuations are imprinted on $\mathrm{CMB}$ at the last scattering surface.

All these effects are readily calculable as they depend only on the geometry and the speed of sound. There are small corrections for the speed of sound near last scattering due to the baryon loading (baryons are relatively more important as the CMB is cooled by the expansion of the universe) and also the effect of the dark matter and even a small correction for dark energy. One finds that the height of the first peak is proportional to the total matter content of the universe while the second (rarefaction) peak is set more by the baryon content and so on. As a result, by carefully fitting to the amplitudes and $l$ values, one can precisely constrain the physical density of baryons and dark matter as well as the geometry of space-time.

The first acoustic peak occurs at $l \sim 200$ or about $0.9^{\circ}$ angular scale for a flat universe. The early observations quickly confirmed this value indicative of a universe near flat. Now observations are getting sufficiently precise that one must take into account the small corrections that come from the possible variation of the contents of the universe. One must take into account that the photon-baryon fluid is not purely photon dominated at the last scattering surface. Baryons add to the mass of the photon-baryon plasma without adding pressure. Also the universe is not exactly matter dominated and there is some dark energy content. The dark energy content comes to dominate in later epochs and changes the distance to the last scattering surface somewhat and thus changes the apparent angles on the sky a small amount. All of these effects are small but are important at the percent to few percent level. By far the most dominant factor is the geometry of the universe and via general relativity the total energy content of the universe is very close to the critical value. In current models this means $\Omega_{\text {total }}=\Omega_{m}+\Omega_{\Lambda}=1.02 \pm 0.02$.

\section{The dark matter and the baryon content of the universe}

By the epoch of last scattering the energy density in dark matter gets to be larger than the energy density in 
photons. As a result, the inertia and gravitational potential of matter is the source of the restoring force (gravity) for the acoustic oscillations and thus affects the amplitude of the oscillations directly. Thus the height of the acoustic peaks is proportional to the total mass density which is primarily dark matter. By measuring the height of the peaks, particularly the first peak, the physical density of matter $\Omega_{m} h^{2}$ is well determined.

Baryons (or ordinary matter) load down the photonbaryon plasma and add inertial (and gravitational) mass to the oscillating system. The effect on the acoustic peaks is easy to understand. If one adds mass to a spring and lets it fall in the gravitational field, with more mass loading the spring, it falls further before pulled back by the spring. Then it rebounds to the same position it started from. That is the maximum compression increases but the rarefaction is unchanged.

Since the odd numbered (first, third, fifth, etc.) acoustic peaks are associated with how much the plasma compresses (falls into the gravitational potential wells), they are enhanced by an increase in the amount of baryons in the universe. The even numbered peaks (second, fourth, sixth) are associated with how far the plasma rarefies (rebounds in the gravitational field). The addition of baryons enhances the odd peaks over the even peaks. Added baryons make the first acoustic peak much larger than the second. The more baryons the more the second peak is relatively suppressed.

If baryons contribute a negligible amount of mass to the plasma, the CMB temperature at the bottom of the potential well oscillates symmetrically around zero. With more baryons in the system, the plasma is loaded down. The plasma compresses further inside the potential well before pressure can reverse the motion. The oscillation is now asymmetric in that the extrema that represent compressions inside potential wells are increased over those that represent rarefactions. The power spectrum does not care about the sign and so takes the absolute value of the temperature fluctuation. Now we see that the first and third peaks are enhanced over the second peak. When we do the full calculation of the power spectrum, the basic physics of a mass on the spring appears as advertised. The odd numbered acoustic peaks in the power spectrum are enhanced in amplitude over the even numbered ones as we increase the baryon density of the universe.

There are second order effects. Since adding mass to a spring slows the oscillation down, adding baryons to the plasma decreases the frequency of the oscillations pushing the position of the peaks to slightly higher multipoles l. (More bayons means slightly slower sound speed so a given number of oscillation cycles must take place over a small size; $\nu_{s} \downarrow \Rightarrow l \uparrow$.) Baryons also affect how sound waves damp, which affects how the spectrum falls off at high $l$. The many ways that baryons show up in the angular power spectrum provides many independent checks on the baryon density of the universe. The baryon density is a quantity that the $\mathrm{CMB}$ can measure to exquisite precision. The $\mathrm{CMB}$ observations agree with big bang nucleosynthesis (BBN) at about $4.4 \pm 0.4 \%$ of critical density. The BBN estimate comes from nuclear physics and conditions in the first few minutes of the big bang. The CMB acoustic peaks ratio comes from atomic physics at 380000 years after the big bang. As a consequence there has been no significant change in the baryon-photon ratio from an energy scale of an $\mathrm{MeV}$ to an $\mathrm{eV}$ and probably to now with an energy scale of $1 / 4000 \mathrm{eV}$ which is a substantial portion of the expansion history of the universe.

\section{Other cosmological parameters including dark energy, equation of state}

The CMB anisotropies are sensitive in varying degrees to a number of other cosmological quantities including the dark energy density, its equation of state, the age of the universe, the optical depth to the reionization of the universe, and the slight tilt of the primordial perturbation spectrum. For some of these observations of the $\mathrm{CMB}$ the angular power spectrum turn out to be extremely sensitive for some others there are degeneracies or near degeneracies that require the $\mathrm{CMB}$ observations to be combined with other cosmological observations to get a truly accurate result. Needless to say, the CMB anisotropies do provide a substantial amount of information. In principle, measuring the power spectrum multipoles provides up to 3000 independent numbers; while we believe that our standard model of cosmology can be well described by less than 20 parameters. Thus we have substantial redundancy going from millions of pixels in our maps, by assuming rotational invariance down to about 3000 numbers in the angular power spectrum and then by cosmological model fitting down to a couple of dozen parameters.

\section{Wilkinson microwave anisotropy probe}

The Wilkinson microwave anisotropy probe (WMAP) mission was the second, more extensive satellite-borne CMB project also by NASA, which followed the COBE (see Fig. 16). The skymap data taken by WMAP have 45 times the sensitivity and 33 times the angular resolution of the COBE DMR mission. WMAP used five separate frequency bands from 23 to $94 \mathrm{GHz}$. The goal of WMAP was to map the relative $\mathrm{CMB}$ temperature over the full sky with an angular resolution $\sim 0.3^{\circ}$, a sensitivity of $20 \mu \mathrm{K}$ per $0.3^{\circ} \times 0.3^{\circ}$ square pixel, with systematic artifacts limited to $5 \mu \mathrm{K}$ per pixel. In February 2003, the WMAP first-year data and results were released and three-year data were released in March 2006 (http:map.gsfc.nasa.gov/). The results strongly support the inflationary big bang models. WMAP also set tight constraints on the cosmological models, among which the $\Lambda \mathrm{CDM}$ model (nearly flat universe with dark energy $\sim 70 \%$, cold dark matter $\sim 25 \%$, baryonic matter $\sim 5 \%$ ) fits best with WMAP along with various independent experiments (see Table V and Fig. 17). 


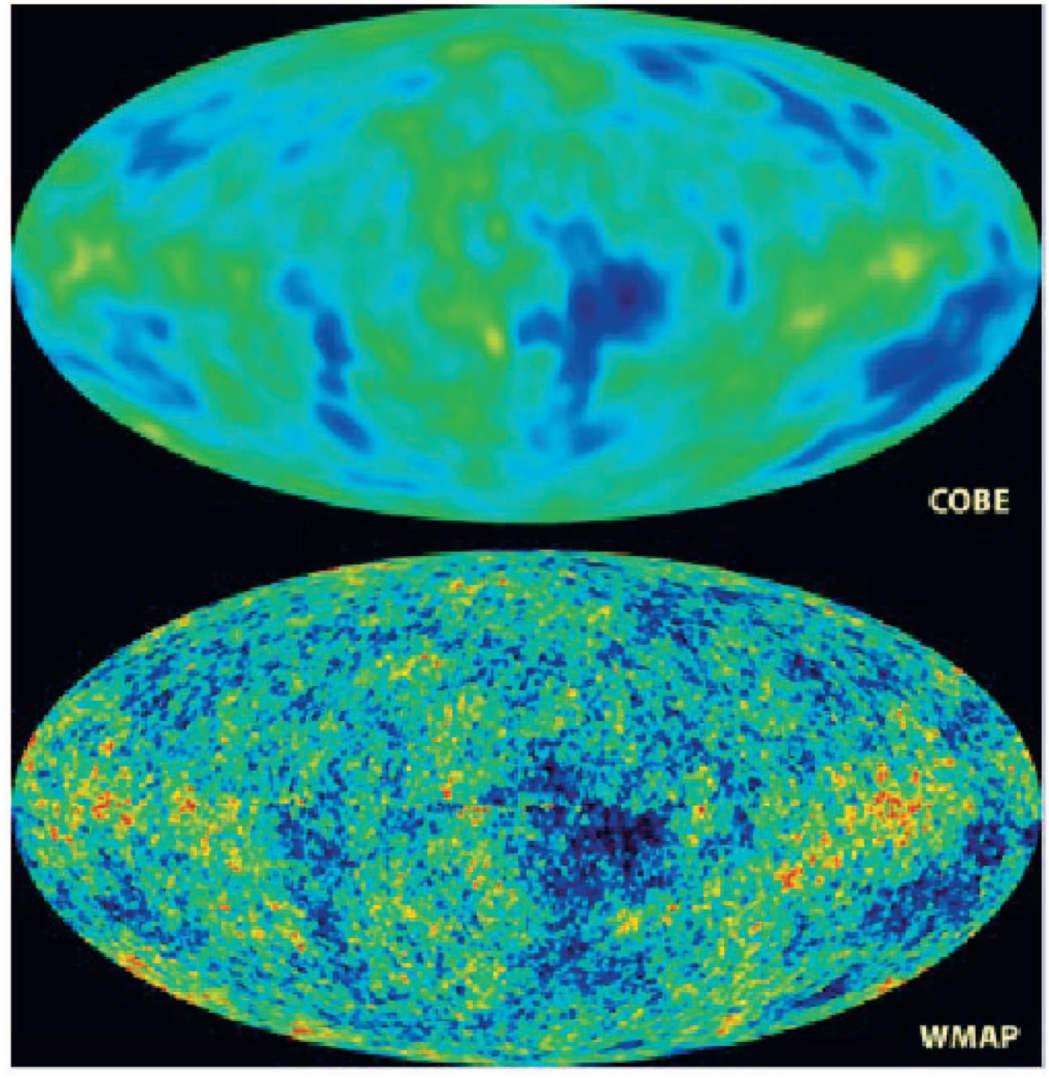

FIG. 16. (Color) A visual comparison of COBE DMR and WMAP. The COBE was able to show only the superhorizon scale $\left(\sim 10^{\circ}\right)$ structures while the WMAP has sufficient angular resolution to show more detailed structures down to subhorizon scale $\left(\sim 0.3^{\circ}\right)$.

\section{PLANCK: The third generation CMB project}

The Planck mission is the third generation space mission for the CMB experiments (see Fig. 18). Conducted by the European Space Agency (ESA), it is scheduled to be launched in 2008. In 1992, two potential space-based CMB experiments, COBRAS and SAMBA, were proposed to ESA and adopted as a combined mission called COBRAS/SAMBA. This project was later renamed as Planck in honor of the German physicist Max Planck. The Planck mission will sweep the full sky with frequency windows from 30 to $857 \mathrm{GHz}$, mapping precise and extensive $\mathrm{CMB}$ anisotropies with angular resolution down to $5^{\prime}$ and sensitivity $\Delta T / T \sim 2 \times 10^{-6}$ [Planck Science Team, 2005, ESA-SCI (2005)1-V2]. Planck will improve on WMAP with advanced features, ten times the sensitivity, two or three times better angular resolution, and six times the frequency coverage of WMAP. The resolutions of WMAP and Planck are visually compared in Fig. 19. Planck measurements are expected to set constraints on physics at energies greater than $10^{15} \mathrm{GeV}$ and its precise measurements of the CMB anisotropy with angular resolution of $10^{\prime}$ will revolutionize cosmology.

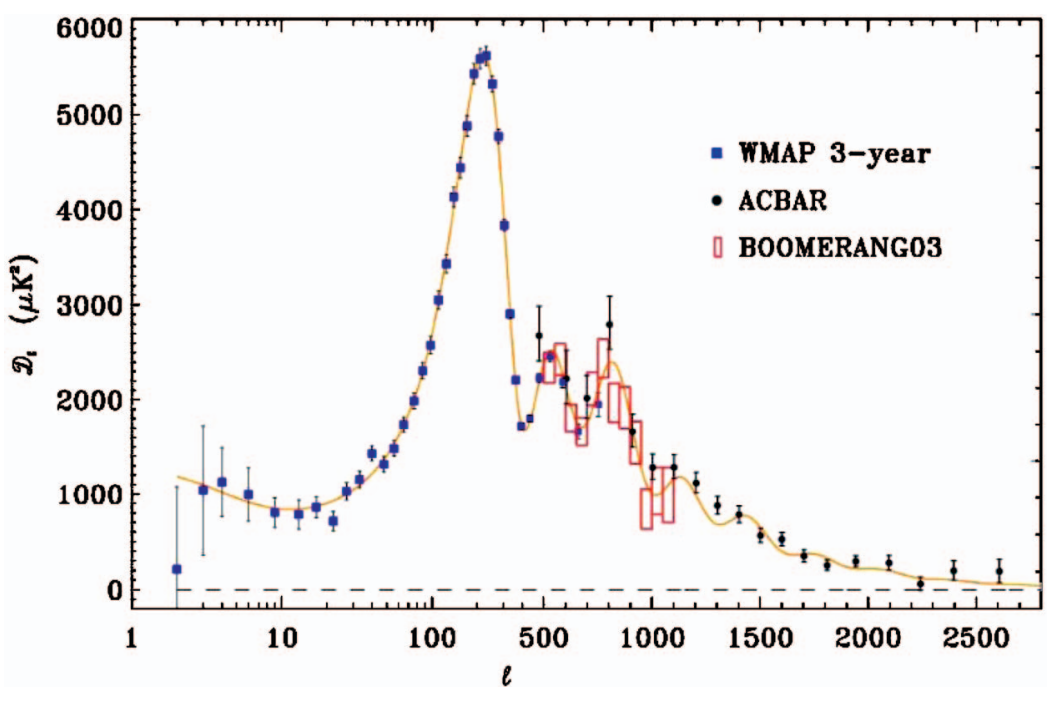

FIG. 17. (Color) Power spectrum predicted by $\Lambda$ CDM model and plots by WMAP 3 -year data experiments, ACBAR and BOOMERANG03. Solid (yellow) curve is the predicted power spectrum of $\Lambda \mathrm{CDM}$ model. 


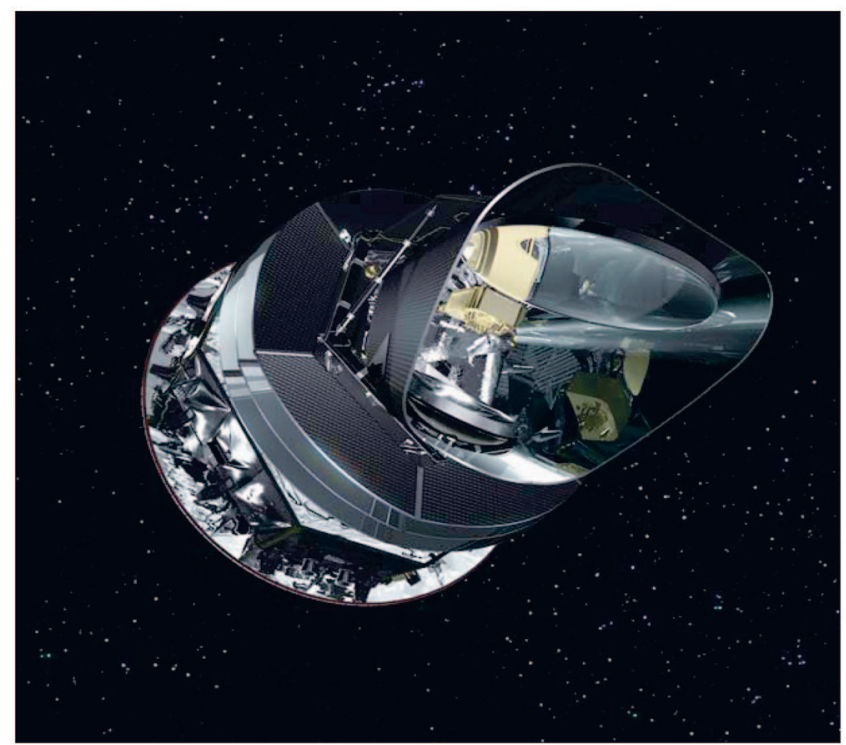

FIG. 18. (Color) An artist's rendering of the Planck satellite in space (http://www.rssd.esa.int/planck/).

\section{CONCLUDING REMARKS}

The demonstration that we have understood the CMB as a relic from the early universe in a simple configuration has led us toward fulfilling the promise that its existence and small deviations are a unique probe of cosmology propelling us into an era which we now call "precision cosmology." It is that precision that makes cosmology a true physical science and now promises to provide a means to know the universe better and to test our very assumptions. The soon to arrive era of the Planck mission, with its related ground-based CMB observations along with other cosmological observations, promises to move us to the $1 \%$ or better level on all key cosmological parameters. As one reaches this level of precision with cross-constraining observations one not only determines the parameters of the universe but also strongly tests the assumptions one has made in determining those parameters.

The cosmic microwave background has many more features yet to be explored fully, and one of the most important topics is the $\mathrm{CMB}$ polarization. Another key topic is the Sunyaev-Zel'dovich effect via clusters of galaxies. Linear polarization of the $\mathrm{CMB}$ arises via $\mathrm{CMB}$ photon scattering with free electrons as long as there is a net quadrupole anisotropy seen by the free electrons. So as Rees predicted in 1968, linear polarization should arise from the anisotropies produced by the primordial perturbations that eventually lead to large scale structure formation. Since Thomson scattering of an anisotropic radiation field also generates linear polarization, the $\mathrm{CMB}$ is predicted to be polarized at the roughly $5 \%$ level ( $\mathrm{Hu}$ and White, 1997). This polarization has been observed by the DASI (Kovac et al., 2002; Leitch et al., 2005) group and a few following instruments. In 2003 the WMAP experiment demonstrated that it was able to measure the TE cross-correlation power spectrum (Kogut et al., 2003). The cross-correlation power spectrum provides supporting evidence of the adiabatic nature of the perturbations, as well as directly constraining the thickness of the last scattering surface. Since the polarization anisotropies are generated in this scattering surface, the existence of correlations at angles far larger than about a degree demonstrate that there were superHubble horizon length fluctuations at the recombination epoch. We are just now in the early phases of observing and exploiting the polarization of the CMB.

However, polarization of the CMB can arise from various sources not yet observed such as rescattering of the $\mathrm{CMB}$ photons during the reionization and gravitational waves from inflation and small scale anisotropies. Polarization measurements are anticipated to significantly improve the accuracy of cosmological parameters and measurements will, in addition to temperature anisotropy measurements, provide an independent test for cosmological models. The $B$ mode (due to gravitational waves) polarization in particular will give substantial in-

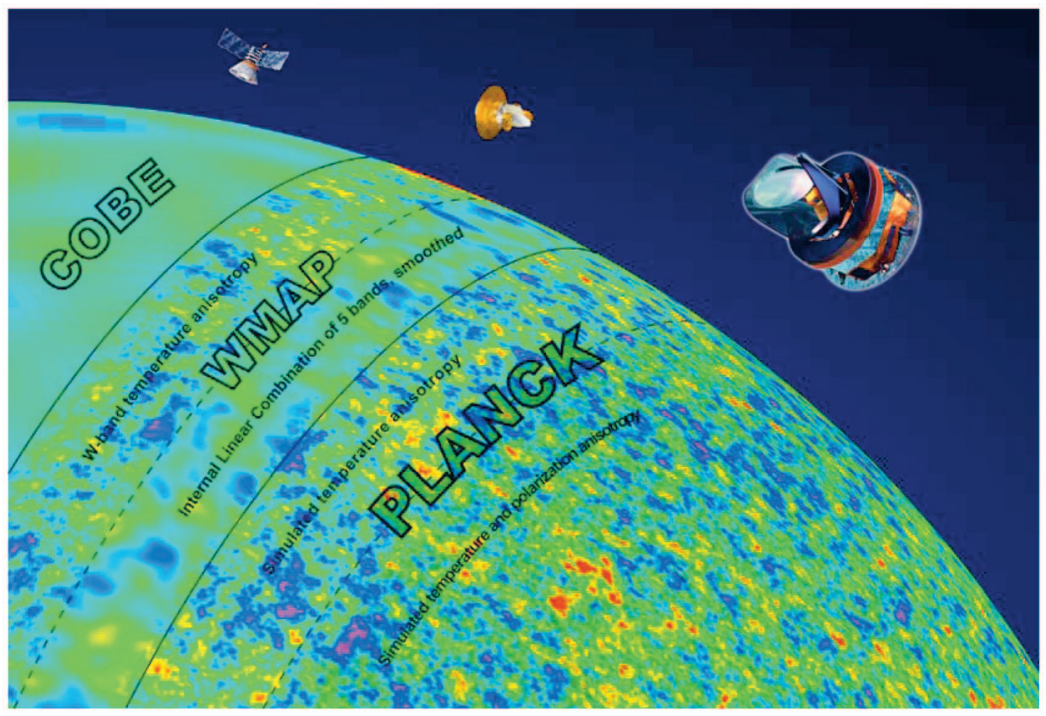

FIG. 19. (Color) The progression of satellite $\mathrm{CMB}$ map resolutions going from the COBE DMR discovery maps to the WMAP and anticipated Planck map (http://www.rssd.esa.int/ planck/). 
formation on the energy scale of inflation. The CMB is also a favored subject in which one can probe theorized remnants of big bang inflation such as topological defects (http://cosmos.lbl.gov/) which are directly related to physics in very early universe $\left(T \sim 10^{16} \mathrm{GeV}\right)$.

\section{ACKNOWLEDGMENTS}

I would like to thank and acknowledge the many people who contributed to this work, particularly my many colleagues and co-workers over the years and particularly the COBE team. The development of cosmology has been a significant intellectual achievement powered by the work of very many scientists, engineers, and staff over many decades. A number of slides and figures that I used in my Nobel Lecture were provided by others and many names were cut off; unfortunately, in the rush of connecting my laptop, the wrong resolution was used and the edges of the slides not projected. Among the many slides were those from Don Groom, Al Kogut, Wayne Hu, Ned Wright, WMAP+SDSS, Max Tegmark, David Wilkinson, Julian Borrill, and others which were there but not shown. I thank a number of colleagues, especially my graduate students and post docs for their discussions and comments both at present and over the many years as well as for their great excitement and intellectual efforts on the various projects. Eunhwa Jeong and Bruce Grossan were directly helpful with this write up. This work was supported by the Lawrence Berkeley National Laboratory and the Department of Physics at the University of California, Berkeley.

\section{REFERENCES}

Alpher, R. A., H. Bethe, and G. Gamow, 1948, "The origin of chemical elements," Phys. Rev. 73, 803-804.

Alpher, R. A., J. W. Follin, and R. C. Herman, 1953, "Physical conditions in the initial stages of the expanding universe," Phys. Rev. 92, 1347-1361.

Alpher, R. A., G. Gamow, and R. Herman, 1967, "Thermal cosmic radiation and the formation of protogalaxies," Proc. Natl. Acad. Sci. U.S.A. 58, 2179-2186.

Alpher, R. A., and R. C. Herman, 1953, "The origin and abundance distribution of the elements," Annu. Rev. Nucl. Sci. 2 , $1-40$.

Alpher, R. A., and R. C. Herman, 1988, "Reflections on early work on 'big bang' cosmology," Phys. Today 41 (8), 24.

Alpher, R. A., and R. Herman, 1990, "Early work on "bigbang' cosmology and the cosmic blackbody radiation," Modern Cosmology in Retrospect, edited by B. Bertotti, R. Balbinot, and S. Bergia (Cambridge University Press, Cambridge, England), p. 129.

Alpher, R. A., R. Herman, and G. A. Gamow, 1948, "Thermonuclear reactions in the expanding universe," Phys. Rev. 74, 1198.

Bahcall et al., 1973, Astrophys. J., Lett. Ed. 182, L95.

Bensadoun et al., 1993, Astrophys. J. 409, 1.

Bernstein et al., 1990, Astrophys. J. 362, 107.

Bersanelli et al., 1989, Astrophys. J. 339, 632.

Bersanelli et al., 1994, Astrophys. J. 424, 517.

Birkinshaw, M., 1999, Phys. Rep. 310, 98.
Boggess, N. W., et al., 1992, "The COBE mission: Its design and performance two years after launch," Astrophys. J. 397, 420-429.

Boughn, S. P., E. S. Cheng, and D. T. Wilkinson, 1981, "Dipole and quadrupole anisotropy of the $2.7 \mathrm{~K}$ radiation," Astrophys. J., Lett. Ed. 243, L113-L117.

Boynton and Stokes, 1974, Nature (London) 247, 528.

Boynton, P. E., R. A. Stokes, and D. T. Wilkinson, 1968, "Primeval fireball intensity at $\lambda=3.3 \mathrm{~mm}$," Phys. Rev. Lett. 21, 462.

Carlstrom, J., M. Joy, L. Grego, G. Holder, W. L. Holzapfel, S. LaRoque, J. J. Mohr, and E. D. Reese, 2000, The SunyaevZel'dovich effect: Results and future prospects, IAP Conference Proceedings.

Carlstrom, J. E., G. P. Holder, and E. D. Reese, 2002 Annu. Rev. Astron. Astrophys. 40, 643.

Cheng, E. S., P. R. Saulson, D. T. Wilkinson, and B. E. Corey, 1979, "Large-scale anisotropy in the $2.7 \mathrm{~K}$ radiation," Astrophys. J., Lett. Ed. 232, L139-L143.

Conklin, E. K., and R. N. Bracewell, 1967, Phys. Rev. Lett. 18, 614.

Crane et al., 1986, Astrophys. J. 309, 822.

Crane et al., 1989, Astrophys. J. 346, 136.

Danese, L., and G. de Zotti, 1977, "The relic radiation spectrum and the thermal history of the Universe," Riv. Nuovo Cimento 7 (2), 277-362.

Danese, L., and G. F. De Zotti, 1982, "Double compton process and the spectrum of the microwave background," Astron. Astrophys. 107, 39.

Davis, R. L., H. M. Hodges, G. F. Smoot, P. J. Steinhardt, and M. S. Turner, 1993, "Cosmic microwave background probes models of inflation," Phys. Rev. Lett. 69, 1856-1859.

De Amici et al., 1985, Astrophys. J. 298, 710.

De Amici et al., 1991, Astrophys. J. 381, 341.

Dicke, R. H., and P. J. Peebles, 1965, Space Sci. Rev. 4, 419.

Dicke, R. H., P. J. E. Peebles, P. S. Roll, and D. T. Wilkinson,

1965, “Cosmic black-body radiation,” Astrophys. J. 142, 414.

Doroshkevich, A. G., and I. G. Novikov, 1964, Sov. Phys.

Dokl. 9, 11.

Ewing et al., 1967, Phys. Rev. Lett. 19, 1251.

Fackler, O., D. Frisch, J. Martin, G. Smoot, and L. Sompayrac, 1973 , "Test of the $\Delta S=\Delta Q$ rule in Ke3 decay," Phys. Rev. Lett. 31, 847-850.

Fixsen, D. J., E. S. Cheng, and D. T. Wilkinson, 1983, "Largescale anisotropy in the $2.7 \mathrm{~K}$ radiation with a balloon-borne maser radiometer at 24.5 GHz,” Phys. Rev. Lett. 50, 620-622. Fixsen, D. J., A. Kogut, S. Levin, M. Limon, P. Lubin, P. Mirel, M. Seiffert, and E. Wollack, 2004, "The temperature of the CMB at $10 \mathrm{GHz}$," Astrophys. J. 612, 86-95. The measured radiometric temperature of the $\mathrm{CMB}$ is $2.721+/-0.010 \mathrm{~K}$ at $10 \mathrm{GHz}$ and $2.694+/-0.032 \mathrm{~K}$ at $30 \mathrm{GHz}$.

Fixsen, D. J., et al., 1996, Astrophys. J. 473, 576.

Friedmann, A. A., P. Boynton, F. Melchiori, and G. F. Smoot, 1980, "A possible 4-way stretch in the universe," Sci. News (Washington, D. C.) 117, 54.

Ge et al., 1997, Astrophys. J. e-print arXiv:astro-ph/9607145. Gorenstein, M. V., R. A. Muller, G. F. Smoot, and A. Tyson, 1978, "Radiometer system to map the cosmic background radiation," Rev. Sci. Instrum. 49, 4.

Gorenstein, M. V., and G. F. Smoot, 1981, "Large-angular-scale anisotropy in the cosmic background radiation," Astrophys. J. 244, 361-381. Results of an extended series of airborne measurements of large-angular-scale anisotropy in the $3-\mathrm{K}$ 
cosmic background radiation are reported. A dual-antenna microwave radiometer operating at $33 \mathrm{GHz}$ flown aboard a U-2 aircraft to $20-\mathrm{km}$ altitude on 11 flights between December 1976 and May 1978 measured differential intensity between pairs of directions distributed over most of the northern hemisphere. Measurements show clear evidence of anisotropy that is readily interpreted as due to the solar motion relative to the sources of the radiation. The anisotropy is well fitted by a first order spherical harmonic of amplitude $3.6+$ or $-0.5 \mathrm{mK}$, corresponding to a velocity of $360+$ or $-50 \mathrm{~km} / \mathrm{s}$ toward the direction $11.2+$ or $-0.5 \mathrm{~h}$ of right ascension and $19^{\circ}+$ or $-8^{\circ}$ declination.

Harrison, E. R., 1970, "Fluctuations at the threshold of classical cosmology,” Phys. Rev. D 1, 2726.

Hawking, S. W., 1969, "On the rotation of the universe," Mon. Not. R. Astron. Soc. 142, 129-141.

Hawking, S. W., and G. F. R. Ellis, 1968, "The cosmic blackbody radiation and the existence of singularities in our universe," Astrophys. J. 152, 25.

Howell and Shakeshaft, 1966, Nature (London) 210, 1318.

Howell and Shakeshaft, 1967a, Nature (London) 216, 7.

Howell and Shakeshaft, 1967b, Nature (London) 216, 753.

$\mathrm{Hu}, \mathrm{W}$., and S. Dodelson, 2002, Annu. Rev. Astron. Astrophys. 40, 171.

Hu, W., and M. White, 1997, Astrophys. J. 435, 568.

Janssen, M. A., S. M. Bednarczyk, S. Gulkis, H. W. Marlin, and G. F. Smoot, 1979, "Pattern measurements of a low-sidelobe horn antenna," IEEE Trans. Antennas Propag. AP-27, 551555 .

Jarosik, N., et al., 2006, "Three-year Wilkinson microwave anisotropy probe (WMAP) observations: Beam profiles, data processing, radiometer characterization and systematic error limits," e-print arXiv:astro-ph/0603452.

Johnson, D. G., and D. T. Wilkinson, 1987, "A 1\% measurement of the temperature of the cosmic microwave radiation at $\lambda=1.2$ centimeters," Astrophys. J., Lett. Ed. 313, L1-L4.

Kaiser and Wright, 1990, Astrophys. J. Lett. 356, L1.

Keegstra, P. B., G. F. Smoot, K. M. Gorski, G. Hinshaw, and L. Tenorio, 1997, Generalized Spherical Harmonics for All-Sky Polarization Studies Astronomical Data Analysis Software and Systems VI, A.S.P. Conference Series, Vol. 125, edited by Gareth Hunt and H. E. Payne, p. 198.

Kislyakov et al., 1971, Sov. Astron. 15, 29.

Kogut, A., A. J. Banday, C. L. Bennett, K. M. Gorski, G. Hinshaw, P. D. Jackson, P. Keegstra, C. Lineweaver, G. F. Smoot, L. Tenorio, and E. L. Wright, 1996, "Calibration and systematic error analysis for the COBE DMR 4 year sky maps," Astrophys. J. 470, 653.

Kogut et al., 1988, Astrophys. J. 325, 1.

Kogut et al., 1990, Astrophys. J. 355, 102.

Kogut, A., et al., 2003, Astrophys. J. S148, 161.

Kovac, J. M., E. M. Leitch, C. Pryke, J. E. Carlstrom, N. W. Halverson, and W. L. Holzapfel, 2002, "Detection of polarization in the cosmic microwave background using DASI," Nature (London) 420, 772-787.

Leitch, E. M., J. M. Kovac, N. W. Halverson, J. E. Carlstrom, C. Pryke, and M. W. E. Smith, 2005, "Degree angular scale interferometer 3 year cosmic microwave background polarization results," Astrophys. J. 624, 10-20.

Levin et al., 1988, Astrophys. J. 334, 14.

Levin et al., 1992, Astrophys. J. 396, 3.

Lu, Sargent, Womble, and Barlow, 1995, e-print.

Lubin, P. M., G. L. Epstein, and G. F. Smoot, 1983a, “3-mm anisotropy measurement and the quadrupole component in the cosmic background radiation," Phys. Rev. Lett. 50, 616.

Lubin, P., G. L. Epstein, and G. F. Smoot, 1983b, "Four-way stretch universe denied," Sci. News (Washington, D. C.) 123, 126.

Lubin, P., P. Melese, and G. F. Smoot, 1983, "Linear and circular polarization of the cosmic background radiation," Astrophys. J., Lett. Ed. 273, L51-L54.

Lubin, P. M., and G. F. Smoot, 1979, "Search for linear polarization of the cosmic background radiation," Phys. Rev. Lett. 42, 129-132.

Lubin, P., T. Villela, G. Epstein, and G. F. Smoot, 1985, "A map of the cosmic background radiaton at 3 millimeters," Astrophys. J., Lett. Ed. 298, L1-L5. Data from a series of balloon flights covering both the northern and southern hemispheres, measuring the large angular scale anisotropy in the cosmic background radiation at $3.3 \mathrm{~mm}$ wavelength, are presented. The data cover $85 \%$ of the sky to a limiting sensitivity of $0.7 \mathrm{mK}$ per $7^{\circ}$ field of view. The data show a 50 -sigma (statistical error only) dipole anisotropy with an amplitude of $3.44+/-0.17 \mathrm{mK}$ and a direction of alpha $=11.2 \mathrm{~h}+/-0.1 \mathrm{~h}$, and delta $=-6.0^{\circ}+/-1.5^{\circ}$. A $90 \%$ confidence level upper limit of 0.00007 is obtained for the rms quadrupole amplitude. Flights separated by six months show the motion of earth around the sun3. Galactic contamination is very small, with less than $0.1 \mathrm{mK}$ contribution to the dipole quadrupole terms. A map of the sky has been generated from the data.

Mandolesi et al., 1986, Astrophys. J. 310, 561.

Mather, J. C., et al., 1994, "Measurement of the cosmic microwave background spectrum by the COBE FIRAS instrument," Astrophys. J. 420, 439-444.

Meyer and Jura, 1985, Astrophys. J. 297, 119.

Meyer et al., 1986, Astrophys. J., Lett. Ed. 308, L37.

Meyer et al., 1989, Astrophys. J. 343, L1.

Millea et al., 1971, Phys. Rev. Lett. 26, 919.

Nanos, G. P., Jr., 1979, "Polarization of the blackbody radiation at 3.2 centimeters," Astrophys. J. 232, 341-347.

Otoshi and Stelzreid, 1975, IEEE Trans. Instrum. Meas. 24, 174.

Palazzi et al., 1990, Astrophys. J. 357, 14.

Palazzi et al., 1992, Astrophys. J. 398, 53.

Partridge, R. B., 1995, 3K: The Cosmic Microwave Background Radiation, Cambridge Astrophysics Series (Cambridge University Press, Cambridge, England).

Partridge, R. B., and D. T. Wilkinson, 1967, Phys. Rev. Lett. 18, 557.

Peebles, P. J. E., 1971, Physical Cosmology (Princeton University Press, Princeton, NJ).

Peebles, P. J. E., 1993, Principles of Physical Cosmology (Princeton University Press, Princeton, NJ), p. 168.

Peebles, P. J. E., and J. T. Yu, 1970, "Primeval adiabatic perturbation in an expanding universe," Astrophys. J. 162, 815. Pelyushenko and Stankevich, 1969, Sov. Astron. 13, 223.

Penzias, A. A., and R. W. Wilson, 1965, "A measurement of excess antenna temperature at $4080 \mathrm{mc} / \mathrm{s}$," Astrophys. J. 142, 419-421.

Perlmutter, S., et al., 1999, "Measurements of omega and lambda from 42 high redshift supernovae," Astrophys. J. 517, 565-586.

Planck Science Team, PLANCK BLUEBOOK, 2005, available online, http://www.rssd.esa.int/Planck/

Puzanov et al., 1968, Sov. Astron. 11, 905.

Rees, M., 1968, "Polarization and spectrum of the primeval 
radiation in an anisotropic universe," Astrophys. J. 153, L1.

Riess, A. G., et al., 1998, "Observational evidence from supernovae for an accelerating universe and a cosmological constant," Astron. J. 116, 1009-1038.

Roll, P. G., and D. T. Wilkinson, 1966, "Cosmic background radiation at $3.2 \mathrm{~cm}$-support for cosmic black-body radiation," Phys. Rev. Lett. 16, 405.

Roll, P. G., and D. T. Wilkinson, 1967. "Measurement of cosmic background radiation at $3.2 \mathrm{~cm}$ wavelength," Ann. Phys. (N.Y.) 44, 289.

Roth et al., 1993, Astrophys. J. Lett. 413, L67.

Sachs, R. K., and A. M. Wolfe, 1967, "Perturbations of a cosmological model and angular variations of the microwave background," Astrophys. J. 147, 73.

Schuster et al., 1993, Ph.D. thesis (U.C. Berkeley, Berkeley, CA).

Sciama, D. W., 1967, "Peculiar velocity of the sun and the cosmic microwave background," Phys. Rev. Lett. 18, 1065.

Sciama, D. W., 1972, "Eppur si muove," Comments Astrophys. Space Phys. 4, 35.

Seljak, U., and M. Zaldarriaga, 1996, Astrophys. J. 469, 437.

Silk, J., 1967, "1967 fluctuations in the primordial fireball," Nature (London) 215, 1155-1156.

Silk, J., 1968, "Cosmic black-body radiation and galaxy formation," Astrophys. J. 151, 459-471.

Sironi et al., 1990, Astrophys. J. 357, 301.

Sironi et al., 1991, Astrophys. J. 378, 550.

Smoot, G. F., 1980, "Aether drift and the isotropy of the universe: A measurement of anisotropes in the primordial blackbody radiation," Final Report, 1 Nov. 1978-31 Oct. 1980, University of California, Berkeley. Lawrence Berkeley Lab. Large-angular-scale anisotropies in the $3 \mathrm{~K}$ primordial blackbody radiation were detected and mapped with a sensitivity of $2 \times 10^{-4} \mathrm{~K}$ and an angular resolution of about $10^{\circ}$. The motion of the earth with respect to the distant matter of the Universe ("aether drift") was measured and the homogeneity and isotropy of the Universe (the "cosmological principle") was probed. The experiment uses two Dicke radiometers, one at $33 \mathrm{GHz}$ to detect the cosmic anisotropy, and one at $54 \mathrm{GHz}$ to detect anisotropies in the residual oxygen above the detectors. The system was installed in the NASA-Ames earth survey aircraft (U-2), and operated successfully in a series of flights in both the northern and southern hemispheres. Data taking and analysis to measure the anisotropy were successful.

Smoot, G. F., 1994, "COBE observations of the early universe," J. R. Astron. Soc. Can. 88.

Smoot, G. F., 1997, "The CMB anisotropy experiments," e-print arXiv:astro-ph/9705135.

Smoot, G. F., M. Bensadoun, M. Bersanelli, G. de Amici, A. Kogut, S. Levin, and C. Witebsky, 1987, "Long-wavelength measurements of the cosmic microwave background radiation spectrum," Astrophys. J., Lett. Ed. 317, L45-L49.

Smoot, G. F., and K. Davidson, 1993, Wrinkles In Time (Avon Books, New York).

Smoot, G. F., G. de Amici, S. D. Friedman, C. Witebsky, N. Mandolesi, R. B. Partridge, G. Sironi, L. Danese, and G. de Zotti, 1983a, "New multifrequency measurements of the spectrum of the cosmic background radiation general relativity and gravitation," Vol. 1, Classical Relativity, Proceedings of the 10th International Conference on General Relativity and Gravitation, Padova, Italy, edited by B. Bertotti, F. de Felice, and A. Pascolini (Consiglio Nazionale delle Ricerche, Rome), p. 865.

Smoot, G. F., G. de Amici, S. D. Friedman, C. Witebsky, N. Mandolesi, R. B. Partridge, G. Sironi, L. Danese, and G. de Zotti, 1983b, "Low-frequency measurement of the spectrum of the cosmic background radiation," Phys. Rev. Lett. 51, 1099-1102.

Smoot, G. F., G. de Amici, S. D. Friedman, C. Witebski, N. Mandolesi, R. B. Partridge, G. Sironi, L. Danese, and G. de Zotti, 1984, "New multifrequency measurements of the spectrum of the cosmic background radiation," COSPAR, IAU, ESA, et al., Symposium on High-Energy Astrophysics and Cosmology, Pamporovo, Bulgaria, 1983, Adv. Space Res. 3, 465-467.

Smoot, G. F., O. D. Fackler, D. H. Frisch, J. F. Martin, and L. M. Sompayrac, 1975 , " $\mathrm{K}^{0}$ production by $\mathrm{K}^{+}$on platinum at 3 GeV/c,” Nucl. Phys. B 96, 379-400.

Smoot, G. F., S. M. Levin, C. Witebsky, G. de Amici, and Y. Rephaeli, 1988, "An analysis of recent measurements of the temperature of the cosmic microwave background radiation," Astrophys. J. 331, 653-659.

Smoot, G. F., and P. M. Lubin, 1979, "Southern hemisphere measurements of the anisotropy in the cosmic microwave background radiation," Astrophys. J., Lett. Ed. 234, L83-L86. Smoot, G. F., and P. J. Steinhardt, 1993, "Gravity's rainbow," Class. Quantum Grav. 10, S19-S32.

Smoot, G. F., et al., 1977, "Detection of anisotropy in the cosmic blackbody radiation," Phys. Rev. Lett. 39, 898.

Smoot, G., et al., 1990, "COBE differential microwave radiometers-instrument design and implementation," Astrophys. J. 360, 685-695.

Smoot, G. F., et al., 1992, "Structure in the COBE differential microwave radiometer first-year maps," Astrophys. J. Lett. 396, L1-L5.

Smoot, G. F., et al., 1994, "Statistics and topology of the COBE DMR first year maps," Astrophys. J. 437, 1-11.

Songaila et al., 1994a, Nature (London) 368, 599.

Songaila et al., 1994b, Nature (London) 371, 43.

South Pole Telescope, 2007, http://spt.uchicago.edu/; http:// cosmology.berkeley.edu/group/swlh/sp/_telescope/

Spergel, D. N., et al., 2006, "Wilkinson Microwave Anisotropy Probe (WMAP) three year results: Implications for cosmology," e-print arXiv:astro-ph/0603449.

Staggs, S., et al., 1995, "A measurement of the cosmic background spectrum at $1.4 \mathrm{GHz}$," Astrophys. Lett. Commun. 32, 3-6.

Staggs et al., 1996a, Astrophys. J. 458, 407.

Staggs et al., 1996b, Astrophys. J. Lett. 473, L1.

Stankevich et al., 1970, Aust. J. Phys. 23, 529.

Stewart, J. M., and D. W. Sciama, 1967, "Peculiar velocity of the sun and its relation to the cosmic microwave background," Nature (London) 216, 748.

Stokes, R. A., R. B. Partridge, and D. T. Wilkinson, 1967, "New measurements of the cosmic microwave background at $\lambda=3.2 \mathrm{~cm}$ and $\lambda=1.58 \mathrm{~cm}$-evidence in support of a blackbody spectrum," Phys. Rev. Lett. 19, 1199-1202.

Sunyaev, R. A., and Y. B. Zel'dovich, 1970, Astrophys. Space Sci. 7, 3.

Sunyaev, R. A., and Y. Zel'dovich, 1972, Comments Astrophys. Space Phys. 4, 173.

Timbie, P. T., and D. T. Wilkinson, 1990, "A search for anisotropy in the cosmic microwave radiation at medium angular scales," Astrophys. J. 353, 140-144.

Toral, M. A., R. B. Ratliff, M. C. Lecha, J. G. Maruschak, and 
C. L. Bennett, 1989, "Measurements of very low-sidelobe conical horn antennas," IEEE Trans. Antennas Propag. 37, 171-177.

Torres, S., J. Aymon, C. Backus, C. Bennett, S. Gulkis, R. Hollenhorst, D. Hon, Q. H. Huang, M. Janssen, S. Keihm, L. Olson, G. F. Smoot, and E. Wright, 1989, Cosmic Background Explorer/COBE/Satellite Anisotropy Experiment Data Analysis Techniques, Proceedings of the Third International Workshop on Data Analysis in Astronomy, Erice, Italy, edited by V. Di Gesu, L. Scarsi, P. Crane, J. H. Friedman, S. Levialdi, and M. C. Maccarone (Plenum, New York).

Uson, J. M., and D. T. Wilkinson, 1984, "Small-scale isotropy of the cosmic microwave background," Astrophys. J. 283, 471-478.
Uson, J. M., and D. T. Wilkinson, 1985, "Improved limits on small-scale anisotropy in cosmic microwave background," Nature (London), 312, 427-429.

Welch et al., 1967, Phys. Rev. Lett. 18, 1068.

Wilkinson, D. T., 1967, "Measurement of the cosmic microwave background at $8.56 \mathrm{~mm}$ wavelength," Phys. Rev. Lett. 19, 1195-1198.

Wright, E. L., et al., 1992, "Interpretation of the cosmic microwave background radiation anisotropy detected by the COBE differential microwave radiometer," Astrophys. J. Lett. 396, L13-L18.

Zel'dovich, Ya. B., 1972, “A hypothesis, unifying the structure and entropy of the universe," Mon. Not. R. Astron. Soc. 160. 